Pure and Applied Mathematics Quarterly

Volume 8, Number 4

993-1028, 2012

\title{
On the Maslov-type Index for Symplectic Paths with Lagrangian Boundary Conditions and Spectral Flow
}

\author{
Sheng Rao and Xing Lin*
}

\begin{abstract}
In this paper, we consider the Maslov-type index theory for symplectic paths starting from the identity with Lagrangian boundary conditions developed by Chun-gen Liu. We firstly give a brief review and some necessary remarks of this theory and then present an explicit formula describing the connection of this index and the spectral flow. Some new concepts and basic properties of complex symplectic theory are introduced.
\end{abstract}

Keywords: Maslov index; spectral flow; Lagrangian boundary conditions; Hamiltonian system; complex symplectic geometry.

\section{Contents}

0. Introduction 994

1. The Maslov-type index with Lagrangian boundary conditions 996

2. The relation between Lagrangian-index and spectral flow 1002

3. A new index with arbitrary boundary conditions 1016

4. Acknowledgement 1027

$\begin{array}{ll}\text { References } & 1027\end{array}$

Received January 7, 2011

MSC(2010): 53D12, 58J30, 70S05.

${ }^{*}$ Corresponding author 


\section{INTRODUCTION}

Let $\left(\mathbb{R}^{2 n}, \omega_{0}\right)$ be the standard linear symplectic space with $\omega_{0}=\sum_{j=1}^{n} d x_{j} \wedge d y_{j}$. A Lagrangian subspace $L$ of $\mathbb{R}^{2 n}$ is an $n$-dimensional subspace satisfying $\left.\omega_{0}\right|_{L}=0$. The set of all Lagrangian subspaces in $\mathbb{R}^{2 n}$ is denoted by $\operatorname{Lag}(n)$. The linear symplectic group $S p(2 n)$ is the set of linear transformation $\varphi: \mathbb{R}^{2 n} \rightarrow \mathbb{R}^{2 n}$ satisfying $\varphi^{*} \omega_{0}=\omega_{0}$. The matrix $J=\left(\begin{array}{cc}0 & -I_{n} \\ I_{n} & 0\end{array}\right)$ is an example of the linear transformation corresponds to a matrix $M$ satisfying $M^{T} J M=J$. We say a matrix satisfying this condition is a symplectic matrix. In this paper we always denote the symplectic group by

$$
S p(2 n)=\left\{M \in \mathcal{L}\left(\mathbb{R}^{2 n}\right) \mid M^{T} J M=J\right\},
$$

where $\mathcal{L}\left(K^{n}\right)$ denotes the group of all $n \times n$ matrices with entries in the field $K$ under the usual matrix multiplication.

For a Lagrangian subspace $L$ and a matrix $M \in S p(2 n)$, the image $M(L)$ of $L$ transformed by the symplectic action $M$ is still a Lagrangian subspace. We define the $L$-singular subset of $S p(2 n)$ by

$$
S p_{L}^{0}(2 n):=\{M \in S p(2 n) \mid \operatorname{dim} M(L) \cap L>0\} .
$$

It is a 1-co-dimensional subset of $S p(2 n)$. Its complement in $S p(2 n)$ is denoted by

$$
S p_{L}^{*}(2 n):=\{M \in S p(2 n) \mid M(L) \cap L=\{0\}\} .
$$

We denote by

$$
\mathcal{P}(2 n)=\left\{\gamma \in C([0,1], S p(2 n)) \mid \gamma(0)=I_{2 n}\right\},
$$

the set of all continuous symplectic paths starting from identity.

The Maslov index for two Lagrangian paths was defined in [3] and [4]. The other Maslov indices such as algebraic and geometric Maslov index theories for non-degenerate symplectic paths are also contained in these two references. In 2006, in their published paper [11], Y. Long, D. Zhang and C. Zhu studied a Maslov-type index theory for symplectic path with fixed Lagrangian boundary condition $L=L_{0}$ via the intersection theory of a Lagrangian path with the constant Lagrangian path $L=L_{0}$ done by Cappell-Lee-Miller index in their celebrated paper [3]. In 2007, in his paper [6], for a Lagrangian subspace $L$, Chun-gen Liu developed an index theory for any symplectic path $\gamma \in$ 
$\mathcal{P}(2 n)$ with respect to the singular set $S p_{L}^{0}(2 n)$. It assigns a pair of integers $\left(i_{L}(\gamma), \nu_{L}(\gamma)\right) \in \mathbb{Z} \times\{0,1, \cdots, n\}$, corresponding to the symplectic path $\gamma$. We call it the Maslov-type index theory with Lagrangian boundary conditions (for convenience, we denote it by Lagrangian-index for short). Here we define $\nu_{L}(\gamma)$ by $\nu_{L}(\gamma)=\operatorname{dim}(\gamma(1) L) \cap L$, but the definition of $i_{L}(\gamma)$ is more complicated. Roughly speaking, $i_{L}(\gamma)$ is the algebraic intersection number of the symplectic path $\gamma$ with the singularity set $S p_{L}^{0}(2 n)$. The index defined in his papers is used to study of the nonlinear Hamiltonian systems with Lagrangian boundary condition. We refer the interested reader to his another paper [7] (and also the new ${ }^{\dagger}$ references in [9]) for some more examples of studying some nonlinear Hamiltonian systems with a Lagrangian boundary condition via this index theory. Here we note that the methods of defining these two indices are different. (For more details for these indices, we refer to [11] where Y. Long et al. gave a complete study of them.) However, as C. Liu and D. Zhang pointed out on pp. 8 of [9], the indices $\mu_{1}(\gamma)$ and $\mu_{2}(\gamma)$ are essentially special cases of the $L$-index $i_{L}(\gamma)$ for Lagrangian subspaces $L_{0}=\{0\} \times \mathbb{R}^{n}$ and $L_{1}=\mathbb{R}^{n} \times\{0\}$ respectively up to a constant $n$.

Now, let us give a brief summary of our contents. In Section 1, we will review this index theory and some related topics. We firstly restate the definition of the index pair $\left(i_{L_{0}}, \nu_{L_{0}}\right)$ for the Lagrangian subspace $L_{0}=\{0\} \oplus \mathbb{R}^{n} \in \operatorname{Lag}(n)$. Then, we can generalize this index pair to the general case $\left(i_{L}, \nu_{L}\right)$, where $L$ is any linear Lagrangian subspace of $\mathbb{R}^{2 n}$. And also, an important calculation formula for $i_{L_{0}}(\gamma \in \mathcal{P}(2 n))$ is given by $\mathrm{C}$. Liu in Theorem 1.3, which states,

$$
i_{L_{0}}(\gamma)=\sum_{j=1}^{n} E\left(\frac{\theta_{j}(1)-\theta_{j}(0)}{\pi}\right),
$$

where $E(a)=\max \{k \in \mathbb{Z} \mid k<a\}$. The well-defineness of the index pair $\left(i_{L}, \nu_{L}\right)$ is proved. Moreover, we will give a rough review of the indices introduced by Y. Long et al. in [11]. In Section 1, we continue to explore the properties of Lagrangian-indices with $L=L_{0}$ since $L$-index can be reduced to the special case $L_{0}$-index. All of the properties are valid for the general case.

In Section 2, we present our main result about the relationship between Lagrangianindex $i_{L_{0}}(\gamma)$ and the $\left(\Lambda, \Lambda^{\prime}\right)$-index $i_{\tilde{\Lambda}_{0}, \tilde{\Lambda}_{0}}(\tilde{\gamma})$ in Theorem 2.16 .

${ }^{\dagger}$ Our manuscript has been completed earlier than May 2009. 
Theorem 0.1. For any symplectic path $\gamma \in \mathcal{P}(2 n)$, there holds

$$
i_{L_{0}}(\gamma)=i_{\tilde{\Lambda}_{0}, \tilde{\Lambda}_{0}}(\tilde{\gamma})-n
$$

Actually, since the $\left(\Lambda, \Lambda^{\prime}\right)$-index $i_{\tilde{\Lambda}_{0}, \tilde{\Lambda}_{0}}(\tilde{\gamma})$ here is a variant of spectral flow, we present an explicit answer to a question about the relation between Lagrangianindex and spectral flow by an explicit formula. Moreover, we introduce some new concepts of complex symplectic theory analogous to the standard symplectic theory and study some useful fundamental properties of the spectral flow introduced in this paper.

In Section 3, we generalize our main Theorem 0.1 for arbitrary Lagrangian boundary conditions by the methods developed here and some results from Lie theory as follows.

Theorem 0.2. For any symplectic path $\gamma \in \mathcal{P}(2 n)$, there holds

$$
i_{L_{0}}^{L_{1}}(\gamma)=i_{\tilde{\Lambda}_{1}, \tilde{\Lambda}_{0}}(\tilde{\gamma})-n
$$

\section{The Maslov-type index With Lagrangian Boundary CONDitions}

In his papers [6, 7], Chun-gen Liu established an index theory for symplectic paths starting from the identity with a Lagrangian boundary condition and developed various properties of the index theory. In this section we will give a brief review of the definition of the index pair for any symplectic path $\gamma \in \mathcal{P}(2 n)$ with the Lagrangian boundary condition (Lagrangian-index for short). It is concerned about the following linear Hamiltonian system

$$
\left\{\begin{array}{l}
\dot{z}(t)=J B(t) z, \forall z \in \mathbb{R}^{2 n}, \forall t \in[0,1], \\
z(0) \in L, z(1) \in L,
\end{array}\right.
$$

where $B(t), t \in[0,1]$, is a family of continuous symmetric matrix functions and $L$ is a Lagrangian subspace of the standard symplectic space. Suppose that $\gamma(t)$ is the fundamental solution of the linear system $\dot{z}(t)=J B(t) z$, i.e., $\gamma(t), t \in[0,1]$, solves this system and satisfying $\gamma(0)=I_{2 n}$. It assigns a pair of integers

$$
\left(i_{L}(\gamma), \nu_{L}(\gamma)\right) \in \mathbb{Z} \times\{0,1, \cdots, n\},
$$

corresponding to the symplectic path $\gamma$. In this section we will review this index theory and give some details and remarks for [6]. We refer the readers to [6] for 
more details on this index, [9] for C. Liu and D. Zhang's breakthrough on the the Bott-type iteration formulas and some abstract precise iteration formulas of this index theory and its applications, and the wonderful literature [10, 1] for the basic knowledge about this topic.

We start from a special case. Suppose $L=L_{0}=\{0\} \oplus \mathbb{R}^{n} \subset \mathbb{R}^{2 n}$ which is a Lagrangian subspace of the linear symplectic space $\left(\mathbb{R}^{2 n}, \omega_{0}\right)$. Write a symplectic path $\gamma(t)$ in the form:

$$
\gamma(t)=\left(\begin{array}{l}
S(t) V(t) \\
T(t) U(t)
\end{array}\right)
$$

where $S(t), T(t), U(t)$ and $V(t)$ are all $n \times n$ matrices. The $n$ vectors coming from the column of the matrix $\left(\begin{array}{l}V(t) \\ U(t)\end{array}\right)$ are linearly independent and span a Lagrangian subspace of $\left(\mathbb{R}^{2 n}, \omega_{0}\right)$. In particular, at $t=0$, this Lagrangian subspace is $L_{0}$.

For this $L_{0}$, define the following two subspace of $S p(2 n)$ by

$$
S p(2 n)_{L_{0}}^{*}=\{M \in S p(2 n) \mid \operatorname{det} V \neq 0\}
$$

and

$$
S p(2 n)_{L_{0}}^{0}=\{M \in S p(2 n) \mid \operatorname{det} V=0\}
$$

for $M=\left(\begin{array}{l}S V \\ T U\end{array}\right)$.

Since the space $S p(2 n)$ is path connected and the $n \times n$ non-degenerate matrix spaces has two path connected components, one with $\operatorname{det} V>0$ and the other with $\operatorname{det} V<0$, the space $S p(2 n)_{L_{0}}^{*}$ has two path connected components as well. Writing

$$
S p(2 n)_{L_{0}}^{ \pm}=\left\{M \in S p(2 n)_{L_{0}}^{*} \mid \pm \operatorname{det} V>0\right\},
$$

then we have $S p(2 n)_{L_{0}}^{*}=S p(2 n)_{L_{0}}^{+} \cup S p(2 n)_{L_{0}}^{-}$. We call $S p(2 n)_{L_{0}}^{0}$ the $L_{0^{-}}$ degenerate subspace of $S p(2 n)$ and $S p(2 n)_{L_{0}}^{*}$ the $L_{0}$-non-degenerate subspace of $S p(2 n)$. We denote the corresponding symplectic path space by

$$
\mathcal{P}(2 n)_{L_{0}}^{*}=\left\{\gamma \in \mathcal{P}(2 n) \mid \gamma(1) \in S p(2 n)_{L_{0}}^{*}\right\}
$$

and

$$
\mathcal{P}(2 n)_{L_{0}}^{0}=\left\{\gamma \in \mathcal{P}(2 n) \mid \gamma(1) \in S p(2 n)_{L_{0}}^{0}\right\} .
$$

$\gamma \in \mathcal{P}(2 n)_{L_{0}}^{0}$ is called an $L_{0}$-degenerate symplectic path and otherwise, $\gamma \in$ $\mathcal{P}(2 n)_{L_{0}}^{*}$ is called an $L_{0}$-non-degenerate symplectic path. 
Definition 1.1. ([6]) We define the $L_{0}$-nullity of any symplectic path $\gamma \in \mathcal{P}(2 n)$ by

$$
\nu_{L_{0}}(\gamma) \equiv \operatorname{dim}_{\operatorname{ker}_{L_{0}}}(\gamma(1)):=\operatorname{dim} \operatorname{ker} V(1)=n-\operatorname{rankV}(1)
$$

with the $n \times n$ matrix function $V(t)$ defined in (1.1).

Since the $n$ vectors coming from the column of the matrix $\left(\begin{array}{l}V(t) \\ U(t)\end{array}\right)$ span a Lagrangian subspace of $\left(\mathbb{R}^{2 n}, \omega_{0}\right)$, we note that rank $\left(\begin{array}{l}V(t) \\ U(t)\end{array}\right)=n$. So the complex matrix $U(t) \pm \sqrt{-1} V(t)$ is invertible. We define a complex matrix function by

$$
Q(t) \triangleq Q_{\gamma}(t)=[U(t)-\sqrt{-1} V(t)][U(t)+\sqrt{-1} V(t)]^{-1} .
$$

It is easy to see that the matrix $Q(t) \in \mathbb{U}(n)$, the unitary matrix for any $t \in[0,1]$. Actually,

$$
\begin{aligned}
& \left([U(t)-\sqrt{-1} V(t)][U(t)+\sqrt{-1} V(t)]^{-1}\right)^{*}[U(t)-\sqrt{-1} V(t)][U(t)+\sqrt{-1} V(t)]^{-1} \\
= & {\left[U(t)^{T}-\sqrt{-1} V(t)^{T}\right]^{-1}\left[U(t)^{T}+\sqrt{-1} V(t)^{T}\right][U(t)-\sqrt{-1} V(t)][U(t)+\sqrt{-1} V(t)]^{-1} } \\
= & {\left[U(t)^{T}-\sqrt{-1} V(t)^{T}\right]^{-1}\left[U(t)^{T} U(t)+V(t)^{T} V(t)\right][U(t)+\sqrt{-1} V(t)]^{-1} } \\
= & {\left[U(t)^{T}-\sqrt{-1} V(t)^{T}\right]^{-1}\left[U(t)^{T}-\sqrt{-1} V(t)^{T}\right][U(t)+\sqrt{-1} V(t)][U(t)+\sqrt{-1} V(t)]^{-1} } \\
= & I,
\end{aligned}
$$

where $M^{*}$ denotes the complex conjugate of $M^{T}$ and the second and third equalities follow from Lemma 2.30 in [13]. Denote by

$$
M_{+}=\left(\begin{array}{cc}
0 & I_{n} \\
-I_{n} & 0
\end{array}\right), M_{-}=\left(\begin{array}{cc}
0 & J_{n} \\
-J_{n} & 0
\end{array}\right) \quad \text { and } \quad J_{n}=\operatorname{diag}(-1,1, \cdots, 1) .
$$

It is clear that $M_{ \pm} \in S p(2 n)_{L_{0}}^{ \pm}$.

For a path $\gamma \in \mathcal{P}(2 n)_{L_{0}}^{*}$, we first adjoin it with a simple symplectic path starting from $J=-M_{+}$, i.e., we define a symplectic path by

$$
\widetilde{\gamma}(t)= \begin{cases}I \cos \frac{(1-2 t) \pi}{2}+J \sin \frac{(1-2 t) \pi}{2}, & t \in[0,1 / 2] \\ \gamma(2 t-1), & t \in[1 / 2,1] .\end{cases}
$$

Then we choose a symplectic path $\beta(t)$ in $S p(2 n)_{L_{0}}^{*}$ starting from $\gamma(1)$ and ending at $M_{+}$or $M_{-}$according to $\gamma(1) \in S p(2 n)_{L_{0}}^{+}$or $S p(2 n)_{L_{0}}^{-}$, respectively. We now 
define a joint path by

$$
\bar{\gamma}(t)=\beta * \widetilde{\gamma}:= \begin{cases}\widetilde{\gamma}(2 t), \quad t \in[0,1 / 2] \\ \beta(2 t-1), & t \in[1 / 2,1] .\end{cases}
$$

By the definition, we see that the symplectic path $\bar{\gamma}$ starting from $-M_{+}$and ending at either $M_{+}$or $M_{-}$. As above, we define

$$
\bar{Q}(t)=[\bar{U}(t)-\sqrt{-1} \bar{V}(t)][\bar{U}(t)+\sqrt{-1} \bar{V}(t)]^{-1}
$$

for $\bar{\gamma}(t)=\left(\begin{array}{l}\bar{S}(t) \bar{V}(t) \\ \bar{T}(t) \bar{U}(t)\end{array}\right)$. By Lemma 2.1, we can choose a continuous function $\bar{\Delta}(t)$ in $[0,1]$ such that

$$
\operatorname{det} \bar{Q}(t)=e^{2 \sqrt{-1} \bar{\Delta}(t)} .
$$

The above argument easily follows that the number $\frac{1}{\pi}(\bar{\Delta}(1)-\bar{\Delta}(0)) \in \mathbb{Z}$ and it does not depend on the choice of the function $\bar{\Delta}(t)$ (cf. Lemma 2.4).

Definition $1.2([6])$. For a symplectic path $\gamma \in \mathcal{P}(2 n)_{L_{0}}^{*}$, define the $L_{0}$-index by

$$
i_{L_{0}}(\gamma)=\frac{1}{\pi}(\bar{\Delta}(1)-\bar{\Delta}(0))
$$

The index of $L_{0}$-degenerate symplectic path $\gamma \in \mathcal{P}(2 n)_{L_{0}}^{*}$ is described in another way. In (1.2), $Q_{\gamma}(t) \in \mathbb{U}(n)$ for any $t \in[0,1]$ (here the subscript $\gamma$ in $Q_{\gamma}(t)$ is to indicate the dependence of $\left.\gamma\right)$. By the non-degeneracy condition, we have $\operatorname{det} V(1) \neq 0$. Suppose $\lambda_{j}(t)=e^{2 \sqrt{-1} \theta_{j}(t)}$ are the eigenvalues of $Q_{\gamma}(t)$ for $j=1,2, \cdots, n$. C. Liu obtained the following important theorem.

Theorem 1.3 (Theorem 3.6 of [6]). For any symplectic path $\gamma \in \mathcal{P}(2 n)$, by using the notations above, there holds

$$
i_{L_{0}}(\gamma)=\sum_{j=1}^{n} E\left(\frac{\theta_{j}(1)-\theta_{j}(0)}{\pi}\right)
$$

where $E(a):=\max \{k \in \mathbb{Z} \mid k<a\}$.

Remark 1.4. From this theorem, we can find that the Lagrangian-index doesn't have the property of path additivity or thus homotopy invariance by path additivity either (cf. Proposition 2.5). The Maslov index introduced in Subsection 2.2 possesses these two critical properties. Note here $\theta_{j}(0)$ is a multiplication of $\pi$. In particular, we can take it as 0. 
For an $L_{0}$-degenerate symplectic path $\gamma \in \mathcal{P}(2 n)_{L_{0}}^{0}$, its $L_{0}$-index is defined by the infimum of the indices of the nearby non-degenerate symplectic paths.

In the general case, let $L$ be any linear Lagrangian subspace of $\mathbb{R}^{2 n}$. Now we are ready to define the index for any symplectic path $\gamma \in \mathcal{P}(2 n)$ with $L$-boundary conditions. We know that $\operatorname{Lag}(n)=\mathbb{U}(n) / O(n)$, which means that for any linear subspace $L \in \operatorname{Lag}(n)$ there is an orthogonal symplectic matrix $P=\left(\begin{array}{cc}A-B \\ B & A\end{array}\right)$ with $A \pm \sqrt{-1} B \in \mathbb{U}(n)$, the unitary matrix, such that $P L_{0}=L . P$ is uniquely determined by $L$ up to an orthogonal matrix $C \in O(n)$, the orthogonal matrix. It means that for any other choice $P^{\prime}$ satisfying the conditions above, there exists a matrix $C \in O(n)$ such that $P^{\prime}=P\left(\begin{array}{ll}C & 0 \\ 0 & C\end{array}\right)$ (cf. Lemma 2.31 in [13]). We define the conjugated symplectic path $\gamma_{P} \in \mathcal{P}(2 n)$ by $\gamma_{P}=P^{-1} \gamma(t) P$.

Definition 1.5. ([6]) Define the L-nullity of any symplectic path $\gamma \in \mathcal{P}(2 n)$ by

$$
\nu_{L}(\gamma) \equiv \operatorname{dim} \operatorname{ker}_{L}(\gamma(1)):=\operatorname{dim} \operatorname{ker} V_{P}(1)=n-\operatorname{rank} V_{P}(1)
$$

with the $n \times n$ matrix function $V_{P}(t)$ defined as in (1.1) with the symplectic path $\gamma$ replaced by $\gamma_{P}$, i.e.,

$$
\gamma_{P}(t)=\left(\begin{array}{ll}
S_{P}(t) & V_{P}(t) \\
T_{P}(t) & U_{P}(t)
\end{array}\right) .
$$

Remark 1.6. The $L$-nullity $\nu_{L}(\gamma)$ is well-defined. In fact, for another choice of $P^{\prime}$ such that $P^{\prime}=P\left(\begin{array}{ll}C & 0 \\ 0 & C\end{array}\right)$ where $C \in O(n)$, the conjugated symplectic path associated with it is

$$
\gamma_{P^{\prime}}=\left(\begin{array}{cc}
C^{-1} & 0 \\
0 & C^{-1}
\end{array}\right)\left(\begin{array}{l}
S_{P}(t) V_{P}(t) \\
T_{P}(t) U_{P}(t)
\end{array}\right)\left(\begin{array}{ll}
C & 0 \\
0 & C
\end{array}\right)=\left(\begin{array}{l}
C^{-1} S_{P}(t) C C^{-1} V_{P}(t) C \\
C^{-1} T_{P}(t) C C^{-1} U_{P}(t) C
\end{array}\right) .
$$

and $\operatorname{dim} \operatorname{ker} V_{P}(1)=\operatorname{dim} \operatorname{ker} C^{-1} V_{P}(1) C$.

For any $L \in \operatorname{Lag}(n)$, we define the following two subspace of $S p(2 n)$ by

$$
\begin{aligned}
& S p(2 n)_{L}^{*}=\left\{M \in S p(2 n) \mid \operatorname{det} V_{P} \neq 0\right\}, \\
& S p(2 n)_{L}^{0}=\left\{M \in S p(2 n) \mid \operatorname{det} V_{P}=0\right\},
\end{aligned}
$$


where $V_{P}$ is defined by

$$
M_{P}=P^{-1} M P=\left(\begin{array}{l}
S_{P}(t) V_{P}(t) \\
T_{P}(t) U_{P}(t)
\end{array}\right) .
$$

The space $S p(2 n)_{L}^{*}$ has two path connected components as well. If we denote the two components by

$$
S p(2 n)_{L}^{ \pm}=\left\{M \in S p(2 n)_{L}^{*} \mid \pm \operatorname{det} V_{P}>0\right\},
$$

then we have $S p(2 n)_{L}^{*}=S p(2 n)_{L}^{+} \cup S p(2 n)_{L}^{-}$. We call $S p(2 n)_{L}^{0}$ the $L$-degenerate subspace of $S p(2 n)$ and $S p(2 n)_{L}^{*}$ the $L$-non-degenerate subspace of $S p(2 n)$. We denote the corresponding symplectic path space by

$$
\mathcal{P}(2 n)_{L}^{*}=\left\{\gamma \in \mathcal{P}(2 n) \mid \gamma(1) \in S p(2 n)_{L}^{*}\right\}
$$

and

$$
\mathcal{P}(2 n)_{L}^{0}=\left\{\gamma \in \mathcal{P}(2 n) \mid \gamma(1) \in S p(2 n)_{L}^{0}\right\} .
$$

If $\gamma \in \mathcal{P}(2 n)_{L}^{0}$, we call it the $L$-degenerate symplectic path and otherwise, if $\gamma \in \mathcal{P}(2 n)_{L}^{*}$, we call it the $L$-non-degenerate symplectic path.

Definition 1.7. ([6]) For a symplectic path $\gamma \in \mathcal{P}(2 n)$, we define the Lagrangianindex by

$$
i_{L}(\gamma)=i_{L_{0}}\left(\gamma_{P}\right)
$$

Remark 1.8. The Lagrangian-index $i_{L}(\gamma)$ is well-defined. In fact, for anther choice $P^{\prime}$ as in Remark 1.6, the conjugated symplectic path associated it is

$$
\begin{aligned}
\gamma_{P^{\prime}} & \triangleq\left(\begin{array}{l}
S_{P^{\prime}}(t) V_{P^{\prime}}(t) \\
T_{P^{\prime}}(t) U_{P^{\prime}}(t)
\end{array}\right)=\left(\begin{array}{cc}
C^{-1} & 0 \\
0 & C^{-1}
\end{array}\right) \gamma_{P}\left(\begin{array}{ll}
C & 0 \\
0 & C
\end{array}\right) \\
& =\left(\begin{array}{l}
C^{-1} S_{P}(t) C C^{-1} V_{P}(t) C \\
C^{-1} T_{P}(t) C C^{-1} U_{P}(t) C
\end{array}\right) .
\end{aligned}
$$

The associated unitary matrix defined in (1.2) becomes

$$
\begin{aligned}
Q_{\gamma_{P^{\prime}}}(t) & =\left[U_{P^{\prime}}(t)-\sqrt{-1} V_{P^{\prime}}(t)\right]\left[U_{P^{\prime}}(t)+\sqrt{-1} V_{P^{\prime}}(t)\right]^{-1} \\
& =C^{-1}\left[U_{P}(t)-\sqrt{-1} V_{P}(t)\right]\left[U_{P}(t)+\sqrt{-1} V_{P}(t)\right]^{-1} C .
\end{aligned}
$$


2. The Relation Between Lagrangian-Index And SPECtral Flow

\subsection{The definition of spectral flow and its properties.}

Lemma 2.1. Suppose $U(t) \in \mathbb{U}(n), t \in[0,1]$ is a continuous family of unitary matrices. Then there exist continuous functions $\theta_{1}(t), \theta_{2}(t), \cdots, \theta_{n}(t), t \in$ $[0,1]$, such that the eigenvalues of $U(t), \forall t \in[0,1]$ are $e^{\sqrt{-1} \theta_{k}(t)}, k=1,2, \cdots, n$ and $\theta_{1}(t) \leq \theta_{2}(t) \leq \cdots \leq \theta_{n}(t), \forall t \in[0,1]$.

Proof. The proof of this lemma is a little analogous to the ideas of Theorems II.5.1 and II.5.2 in [5]. Here we give the main ideas, which are divided as three steps.

Step 1 For any $s \in[0,1]$, assume that $e^{\sqrt{-1} \theta_{k}^{s}(s)}, k=1,2, \cdots, n$, are the eigenvalues of $\mathrm{U}(\mathrm{s})$. Without loss of generality, we assume $\theta_{1}^{s}(s), \cdots, \theta_{n}^{s}(s) \in$ $(-\pi, \pi]$ and then give a numbering $\theta_{l_{1}}^{s}(s) \leq \cdots \leq \theta_{l_{m}}^{s}(s)$, where $l_{j}$ of $\theta_{l_{j}}^{s}(s)$ denotes the algebraic multiplicity of it and $\sum_{j} l_{j}=n$. Set $V_{k}^{s}=\left\{\theta|| \theta-\theta_{k}^{s}(s) \mid<\pi\right\}$. Then there exists a constant $\delta_{s}$, such that for any $t \in[0,1] \cap\left(s-\delta_{s}, s+\delta_{s}\right)$, the eigenvalues of $\mathrm{U}(\mathrm{t})$ are $e^{\sqrt{-1} \theta_{k}^{s}(t)}, k=1,2, \cdots, n$ and $\theta_{l_{1}}^{s}(t) \leq \cdots \leq \theta_{l_{m}}^{s}(t)$, where $\theta_{k}^{s}(t) \in V_{k}^{s}$.

Take $\varepsilon>0$, such that $\varepsilon<\frac{\theta_{k}^{s}(s)-\theta_{k-1}^{s}(s)}{3}$ if $\theta_{k}^{s}(t)>\theta_{k-1}^{s}(s)$, and $\varepsilon<\frac{\theta_{1}^{s}(s)+2 \pi-\theta_{n}^{s}(s)}{3}$. Then there exits $\delta_{s}^{\prime}$, for any $t \in[0,1] \cap\left(s-\delta_{s}^{\prime}, s+\delta_{s}^{\prime}\right)$, such that

$$
\sigma(U(t)) \cap\left\{e^{\sqrt{-1}\left(\theta_{k}^{s}(s)\right) \pm \varepsilon}\right\}=\emptyset .
$$

Claim There exist $l_{1}, l_{2}, \cdots, l_{m}$ eigenvalues near $e^{\sqrt{-1} \theta_{l_{1}}^{s}(t)}, e^{\sqrt{-1} \theta_{l_{1}+1}^{s}(t)}, \cdots$, $e^{\sqrt{-1} \theta_{l_{1}+l_{2}+\cdots+l_{m-1}+1}(t)}$, i.e., $\left\{e^{\sqrt{-1} \theta} \mid \theta \in\left(\theta_{k}^{s}(s)-\varepsilon, \theta_{k}^{s}(s)+\varepsilon\right)\right\}$ respectively.

In fact, set open set $\Omega \in \mathbb{C}$ with smooth boundary $\partial \Omega$ satisfying $\partial \Omega \cap \sigma(U(t))=$ $\emptyset$. Define a projection by

$$
P_{\Omega}(U(t))=-\frac{1}{2 \pi \sqrt{-1}} \int_{\partial \Omega}(U(t)-z I)^{-1} d z .
$$

By the fact that range $P_{\Omega}(U(t))$ is just the eigenspace of $U(t)$ in $\Omega$ and by continuity of $U(t)$, dim range $P_{\Omega}(U(t))=$ const, we can obtain the claim.

So, if $t \in[0,1] \cap\left(s-\delta_{s}^{\prime}, s+\delta_{s}^{\prime}\right)$, we can get a numbering of the eigenvalues. i.e.,

$$
\theta_{1}^{s}(t) \leq \cdots \leq \theta_{n}^{s}(t)
$$

where $\theta_{1}^{s}(s)=\theta_{2}^{s}(s)=\cdots=\theta_{l_{1}}^{s}(s)$. 
Step $2 \theta_{1}^{s}(t), \cdots, \theta_{n}^{s}(t)$ are continuous.

We need to prove $\theta_{k}^{s}(\tilde{t}) \rightarrow \theta_{k}^{s}(t)$ when $\tilde{t} \rightarrow t$. So near $e^{\sqrt{-1} \theta_{k}^{s}(t)}$, by Step 1 , we also can get a similar numbering and thus the algebraic multiplicities are $l_{1}^{t}, l_{2}^{t}, \cdots l_{j}^{t}$, respectively. By the claim above, we have that for $\varepsilon_{t}>0$, if $|\tilde{t}-t| \ll 1$ such that

$$
\begin{gathered}
\sharp\left\{k|| \theta_{k}^{s}(\tilde{t})-\theta_{1}^{s}(t) \mid<\varepsilon_{t}\right\}=l_{1}^{t}, \\
\sharp\left\{k|| \theta_{k}^{s}(\tilde{t})-\theta_{2}^{s}(t) \mid<\varepsilon_{t}\right\}=l_{2}^{t}, \\
\vdots \\
\sharp\left\{k|| \theta_{k}^{s}(\tilde{t})-\theta_{2}^{s}(t) \mid<\varepsilon_{t}\right\}=l_{j}^{t} .
\end{gathered}
$$

So we can get that $\left|\theta_{l_{k}}^{s}(\tilde{t})-\theta_{l_{k}}^{s}(t)\right|<\varepsilon_{t}$.

$\underline{\text { Step } 3}$ Gluing all the small intervals to get a global continuous numbering.

From above, we can get a continuous numbering in a small neighborhood for each point in $[0,1]$ and then by Lebesgue Lemma, we have a Lebesgue constant $\delta$ such that all the intervals with diameters smaller that $\delta$ contained in some neighborhood that obtained in the last two steps. The global continuity is assured by the local continuity in each neighborhood and the numbering is an easy thing.

Definition 2.2. For a continuous family of unitary matrices $\{U(t) \in \mathbb{U}(n), t \in$ $[0,1]\}$, we define the spectral flow of it by

$$
s f\{U(t), t \in[0,1]\}:=\sum_{k=1}^{n}\left(\left[\frac{\theta_{k}(1)}{2 \pi}\right]-\left[\frac{\theta_{k}(0)}{2 \pi}\right]\right),
$$

where $[a]=\max \{k \in \mathbb{Z} \mid k \leq a\}$.

Our definition is inspired by the spectral flow introduced by M. F. Atiyah, V. K. Patodi and I. M. Singer [2].

Example 2.3. It is well-known that spectral flow describes the net change in the number of negative eigenvalues of the operator family. The counting is done so that each negative eigenvalue which becomes nonnegative contributes +1 , and each nonnegative eigenvalue which becomes negative contributes -1 . Here we assume 
that each $\theta(t)$ in $e^{\sqrt{-1} \theta(t)}$ for every $t \in[0,1]$ belongs to $[-\pi, \pi)$ and then

$$
\begin{aligned}
s f\left\{e^{\sqrt{-1} \theta(t)}, t \in[0,1]\right\} & =\left\{\begin{array}{cc}
1 & \theta(0)<0 \\
0 & \theta(0) \geq 0
\end{array}\right\}-\left\{\begin{array}{cc}
1 & \theta(1)<0 \\
0 & \theta(1) \geq 0
\end{array}\right\} \\
& =\left(-\left[\frac{\theta(0)}{2 \pi}\right]\right)-\left(-\left[\frac{\theta(1)}{2 \pi}\right]\right) \\
& =\left[\frac{\theta(1)}{2 \pi}\right]-\left[\frac{\theta(0)}{2 \pi}\right] .
\end{aligned}
$$

In some sense, Definition 2.2 is a generalization of this example.

Lemma 2.4. Definition 2.2 is valid, i.e., this definition is independent of the choice of $\theta_{k}(t), k=1,2, \cdots, n, \forall t \in[0,1]$.

Proof. Suppose that there exists another choice of $\tilde{\theta}_{k}(t), k=1,2, \cdots, n, \forall t \in$ $[0,1]$ satisfying the conditions of Lemma 2.1. Then for each $k$ and any $t \in[0,1]$, we have some $k^{\prime}$, such that $\theta_{k}(t)=\tilde{\theta}_{k^{\prime}}(t)+2 m_{k}(t) \pi$, and so

$$
\sum_{k=1}^{n} \theta_{k}(t)=\sum_{k^{\prime}=1}^{n} \tilde{\theta}_{k^{\prime}}(t)+2\left(\sum_{k=1}^{n} m_{k}(t)\right) \pi
$$

where $m_{k}(t)$ is some integer. Noting here that for any $t \in[0,1]$,

$$
\sum_{k=1}^{n} m_{k}(t)=\text { constant }
$$

we have

$$
\left[\sum_{k=1}^{n} \frac{\theta_{k}(t)}{2 \pi}\right]=\left[\sum_{k=1}^{n} \frac{\tilde{\theta}_{k}(t)}{2 \pi}\right]+\text { constant } .
$$

Then by the following three useful facts

$$
\left[\sum_{k=1}^{n} \frac{\theta_{k}(t)}{2 \pi}\right]=\left[\sum_{k=1}^{n}\left\{\frac{\theta_{k}(t)}{2 \pi}\right\}\right]+\sum_{k=1}^{n}\left[\frac{\theta_{k}(t)}{2 \pi}\right]
$$

and similarly,

$$
\left[\sum_{k=1}^{n} \frac{\tilde{\theta}_{k}(t)}{2 \pi}\right]=\left[\sum_{k=1}^{n}\left\{\frac{\tilde{\theta}_{k}(t)}{2 \pi}\right\}\right]+\sum_{k=1}^{n}\left[\frac{\tilde{\theta}_{k}(t)}{2 \pi}\right],
$$

where $\{x \in \mathbb{R}\}:=x-[x]$,

$$
\left[\sum_{k=1}^{n}\left\{\frac{\theta_{k}(t)}{2 \pi}\right\}\right]=\left[\sum_{k=1}^{n}\left\{\frac{\tilde{\theta}_{k}(t)}{2 \pi}\right\}\right],
$$


one can obtain

$$
\sum_{k=1}^{n}\left[\frac{\theta_{k}(1)}{2 \pi}\right]-\sum_{k=1}^{n}\left[\frac{\theta_{k}(0)}{2 \pi}\right]=\sum_{k=1}^{n}\left[\frac{\tilde{\theta}_{k}(1)}{2 \pi}\right]-\sum_{k=1}^{n}\left[\frac{\tilde{\theta}_{k}(0)}{2 \pi}\right] .
$$

Proposition 2.5 (Basic properties of spectral flow, see also Proposition 3.1 of $[16])$. Let $U(t) \in \mathbb{U}(n), t \in[0,1]$ is a continuous family of unitary matrix. Then the spectral flow of it has the following basic properties.

(a) (Path additivity) For $t^{\prime} \in[0,1]$, we have

$$
s f\left\{U(t), t \in\left[0, t^{\prime}\right]\right\}+s f\left\{U(t), t \in\left[t^{\prime}, 1\right]\right\}=s f\{U(t), t \in[0,1]\} .
$$

(b) (Reversal) By following the notations in Lemma 2.1, we have

$s f\{U(t), t \in[0,1]\}=-s f\left\{U^{-1}(t), t \in[0,1]\right\}+\sum_{k=1}^{n}\left(\delta\left(\frac{\theta_{k}(1)}{2 \pi}\right)-\delta\left(\frac{\theta_{k}(0)}{2 \pi}\right)\right)$, where $\delta(x \in \mathbb{R})= \begin{cases}1 & x \text { is an integer, } \\ 0 & x \text { is not an integer. }\end{cases}$

(c) (Homotopy invariance) Suppose $U_{s}(t) \in \mathbb{U}(n), s, t \in[0,1]$ is a continuous family of unitary matrix, then

$$
s f\left\{U_{s}(t),(s, t) \in \partial([0,1] \times[0,1])\right\}=0 .
$$

(d) (Zero) If $\operatorname{dim} \operatorname{ker}(U(t)-I) \equiv$ constant, then $\operatorname{sf}\{U(t), t \in[0,1]\}=0$.

(e)(Product) Given a unitary path $\hat{U}(t) \in \mathbb{U}(n), t \in[0,1]$, we have

$$
s f\left\{\hat{U}(t)^{*} U(t) \hat{U}(t), t \in[0,1]\right\}=s f\{U(t), t \in[0,1]\},
$$

where $M^{*}$ denotes the complex conjugate of $M^{T}$.

Proof. (a) follows from the definition of spectral flow 2.2 directly.

(b) It is easy to check that the eigenvalues of $U^{-1}(t)$ are

$$
\left\{e^{-\sqrt{-1} \theta_{k}(t)}, k=1,2, \cdots, n\right\}
$$


for any $t \in[0,1]$. By the definition of spectral flow, we have

$$
\begin{aligned}
& s f\{U(t), t \in[0,1]\} \\
= & \sum_{k=1}^{n}\left(\left[\frac{\theta_{k}(1)}{2 \pi}\right]-\left[\frac{\theta_{k}(0)}{2 \pi}\right]\right) \\
= & \sum_{k=1}^{n}\left(E\left(\frac{\theta_{k}(1)}{2 \pi}\right)+\delta\left(\frac{\theta_{k}(1)}{2 \pi}\right)\right)-\sum_{k=1}^{n}\left(E\left(\frac{\theta_{k}(0)}{2 \pi}\right)+\delta\left(\frac{\theta_{k}(0)}{2 \pi}\right)\right) \\
= & \sum_{k=1}^{n}\left(E\left(\frac{\theta_{k}(1)}{2 \pi}\right)-E\left(\frac{\theta_{k}(0)}{2 \pi}\right)\right)+\sum_{k=1}^{n}\left(\delta\left(\frac{\theta_{k}(1)}{2 \pi}\right)-\delta\left(\frac{\theta_{k}(0)}{2 \pi}\right)\right) \\
= & -\left(\sum_{k=1}^{n}\left(\left[-\frac{\theta_{k}(1)}{2 \pi}\right]-\left[-\frac{\theta_{k}(0)}{2 \pi}\right]\right)\right)+\sum_{k=1}^{n}\left(\delta\left(\frac{\theta_{k}(1)}{2 \pi}\right)-\delta\left(\frac{\theta_{k}(0)}{2 \pi}\right)\right) \\
= & -s f\left\{U^{-1}(t), t \in[0,1]\right\}+\sum_{k=1}^{n}\left(\delta\left(\frac{\theta_{k}(1)}{2 \pi}\right)-\delta\left(\frac{\theta_{k}(0)}{2 \pi}\right)\right),
\end{aligned}
$$

where $E(\cdot)$ is defined as in Theorem 1.3 and the last second equality applies the following useful formula,

$$
E(x)=-[-x]-1, \forall x \in \mathbb{R} .
$$

(c) By the same ideas in the proof of Lemma 2.1, for each point $x \in[0,1] \times[0,1]$, we can get a neighborhood $\Omega$ satisfying the following property.

For any two points $x_{1}$ and $x_{2}$ in $\Omega$, let $e^{\theta_{j}^{1}}, e^{\theta_{j}^{2}}, j=1,2, \cdots, n$, be the eigenvalues of $U_{s}(t)$ at them, respectively and $e^{\theta_{i_{k}}^{*}}$ the eigenvalues with algebraic multiplicity $i_{k}$, where $\sum i_{k}=n$ and ' $*$ ' is chosen as 1 or 2 . Then we have for each $i_{k}$,

$$
\left|\frac{\theta_{i_{k}}^{1}}{2 \pi}-\frac{\theta_{i_{k}}^{2}}{2 \pi}\right| \ll 1
$$

and thus, by Property (a), it follows

$$
s f\left\{U_{s}(t),(s, t) \in \partial(\text { any square in } \Omega)\right\}=0 .
$$

Next, by Lebesgue Lemma, if we set the Lebesgue constant as $\delta$ and choose $m \gg 1$, such that $\frac{1}{m}<\delta$, then each small square $\left[\frac{i}{m}, \frac{i+1}{m}\right] \times\left[\frac{j}{m}, \frac{j+1}{m}\right], i, j=$ $0,1, \cdots, n-1$ belongs to some neighborhood with the property. Furthermore, 
since the global continuity of the eigenvalues of $\theta_{i_{k}}(t)$ is assured by the continuity of $\theta_{i_{k}}(t)$ in each small square (locally), we have

$$
\begin{aligned}
0 & =\sum_{i, j=1}^{m-1} s f\left\{U_{s}(t),(s, t) \in \partial\left(\left[\frac{i}{m}, \frac{i+1}{m}\right] \times\left[\frac{j}{m}, \frac{j+1}{m}\right]\right)\right\} \\
& =s f\left\{U_{s}(t),(s, t) \in \partial([0,1] \times[0,1])\right\}
\end{aligned}
$$

where the last equality follows from Property (a) (Path additivity).

(d) From the assumption, we know that for each $t \in[0,1], U(t) \in \mathbb{U}(n)$ have eigenvalues 1 with the same multiplicity. Without loss of generality, we can assume the other eigenvalues $\theta_{k}$ all belong to $(0,2 \pi)$. By the definition of spectral flow, we find that the sum in (2.1) is zero.

(e) It is easy to check that $U(t)$ and $\hat{U}(t)^{*} U(t) \hat{U}(t)$ have the same eigenvalues. Then we can get the result from the definition of spectral flow directly.

2.2. A Maslov index by spectral flow. In this subsection, we will present a Maslov index by spectral flow, in some sense, which can be viewed as a generalization of the Lagrangian-index. And for other interesting connection between various Maslov indices and spectral flow, we refer to [17, 12, 14, 15] and specially [16], from which we have learned a lot. Above all, we introduce some new concepts and notations for complex symplectic theory similar to the (real) symplectic theory.

Definition 2.6. A complex matrix $M \in \mathcal{L}\left(\mathbb{C}^{m}\right)$ is called a $\tilde{J}_{\text {-symplectic matrix, }}$ if it satisfies

$$
M^{*} \tilde{J} M=\tilde{J}
$$

where

$$
\tilde{J}=\left(\begin{array}{cc}
\sqrt{-1} I_{n_{1}} & 0 \\
0 & -\sqrt{-1} I_{n_{2}}
\end{array}\right), n_{1}+n_{2}=m .
$$

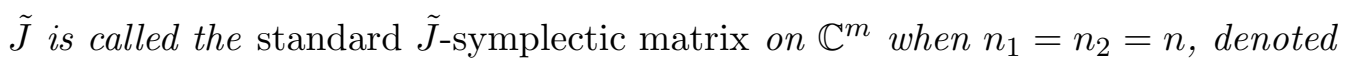
by $\tilde{J}_{0} . I_{n} \in \mathcal{L}\left(\mathbb{R}^{n}\right)$ is the $n \times n$ identity matrix and the zero matrix is denoted by 0 . All the $m \times m \tilde{J}$-symplectic matrices form a subgroup of $\mathcal{L}\left(\mathbb{C}^{m}\right)$ denoted by $S p(\tilde{J})$ and called the $\tilde{J}_{\text {-symplectic group. }}$ 
Firstly, we consider a $2 \times 2$ complex matrix $M=\left(\begin{array}{l}a b \\ c d\end{array}\right)$. By Definition 2.6,

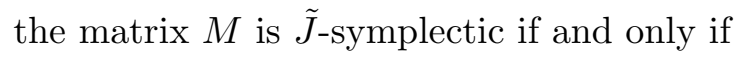

$$
\bar{a} a-\bar{c} c=\bar{d} d-\bar{b} b=1 \text { and } \bar{a} b=\bar{c} d .
$$

Furthermore, if $M$ is unitary, it satisfies

$$
\bar{a} a+\bar{c} c=\bar{d} d+\bar{b} b=1 \text { and } \bar{a} b=\bar{c} d .
$$

In conclusion, $M$ is unitary $\tilde{J}_{\text {-symplectic if and only if }}$

$$
\bar{a} a=\bar{d} d=1 \text { and } \bar{b} b=\bar{c} c=0 \text {, i.e., } \bar{a} a=\bar{d} d=1 \text { and } b=c=0 .
$$

Lemma 2.7. Suppose that a matrix $M \in \mathcal{L}\left(\mathbb{C}^{2 n}\right)$ has the square block form

$$
M=\left(\begin{array}{ll}
A & B \\
C & D
\end{array}\right),
$$

where $A, B, C$ and $D$ are all $n \times n$ matrices. Then, we have

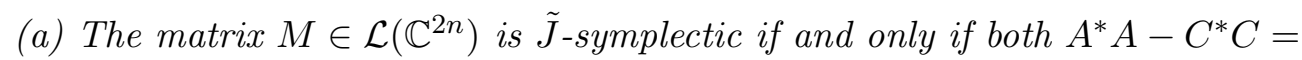
$D^{*} D-B^{*} B=I$ and $A^{*} B=C^{*} D$.

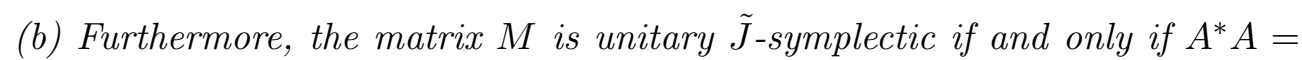
$D^{*} D=I$ and $B=C=0$, i.e., $A, D \in \mathbb{U}(n)$ and $B=C=0$.

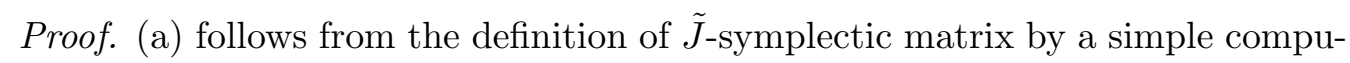
tation.

(b) Furthermore, when the matrix $M$ is unitary, it should further satisfies $A^{*} A+C^{*} C=B^{*} B+D^{*} D=I$ and $A^{*} B+C^{*} D=0$. By (a) we have $A^{*} A=$ $D^{*} D=I$ and $B^{*} B=C^{*} C=0$, i.e., $A^{*} A=D^{*} D=I$ and $B=C=0$.

Definition 2.8. (Definition 4.1 of [16]) Suppose $V$ is a complex vector space of dimension $m$, and $\tilde{\omega}$ is a sesquilinear, complex antisymmetric and non-degenerate bilinear form defined on $V$, i.e., $\tilde{\omega}$ satisfies

i) $\tilde{\omega}(x, y)$ is linear in $x$ and conjugate linear in $y$;

ii) $\tilde{\omega}(u, v)=-\overline{\tilde{\omega}(v, u)}, \forall u, v \in V$;

iii) $\tilde{\omega}(u, v)=0$ for some $u \in V$ and any $v \in V$ implies $u=0$.

Then $\tilde{\omega}$ is called a complex symplectic form on $V$, and $(V, \tilde{\omega})$ is a complex symplectic space. 
Let $(V, \tilde{\omega})$ be a complex symplectic space. $u, v \in V$ are complex symplectically orthogonal, denoted by $u \perp v$, if $\tilde{\omega}(u, v)=0$. For $A, B \subset V$, define $A \perp B$ if $u \perp v$ for all $u \in A$ and $v \in B$. Let $E$ be a linear subspace of $V$. Define

$$
E^{\tilde{\omega}}=\{u \in V \mid \tilde{\omega}(u, v)=0, \forall v \in E\} .
$$

Then $E^{\tilde{\omega}}$ is a linear subspace of $V$, and $\left(E^{\tilde{\omega}}\right)^{\tilde{\omega}}=E$. A linear subspace $E$ of the complex symplectic space $(V, \tilde{\omega})$ is called complex Lagrangian, if $E=E^{\tilde{\omega}}$.

The most popular example is the standard complex symplectic space $\left(\mathbb{C}^{2 n}, \tilde{\omega}\right)$ with $\tilde{\omega}$ defined by the standard $\tilde{J}_{\text {-symplectic matrix }} \tilde{J}_{0}$,

$$
\tilde{\omega}(u, v)=\left\langle\tilde{J}_{0} u, v\right\rangle_{\mathbb{C}}, \forall u, v \in \mathbb{C}^{2 n},
$$

where $\langle u, v\rangle_{\mathbb{C}}$ denotes the standard complex inner product of $u$ and $v$, i.e., $u^{*} v$. Without confusion, we omit the subindex $\mathbb{C}$. The corresponding complex Lagrangian subspaces of this complex symplectic space are called $\tilde{J}_{0}$-Lagrangian subspace and the set of all these subspaces is denoted by $\operatorname{Lag}\left(\tilde{J}_{0}\right)$ for short.

When analogous definitions for complex Lagrangian subspace are adopted here

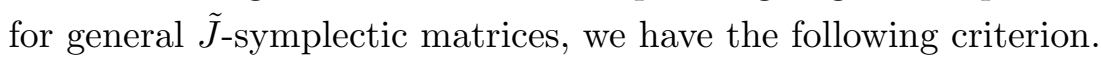

Lemma 2.9. Set $\tilde{J}=\left(\begin{array}{cc}\sqrt{-1} I_{n_{1}} & 0 \\ 0 & -\sqrt{-1} I_{n_{2}}\end{array}\right)$. Then $\Lambda \in \operatorname{Lag}(\tilde{J})$ if and only if there exists a unique unitary map $U: \mathbb{C}^{n_{1}} \rightarrow \mathbb{C}^{n_{2}}$, satisfying

$$
\Lambda=G r(U):=\left\{\left(\begin{array}{c}
x \\
U x
\end{array}\right) \mid x \in \mathbb{C}^{n_{1}}\right\}
$$

and $n_{1}=n_{2}$.

Proof. Sufficiency. This follows from the definition of $\tilde{J}$-Lagrangian subspace by direct computation.

Necessity. From the definition, for any two vectors $\left(\begin{array}{l}x_{1} \\ y_{1}\end{array}\right),\left(\begin{array}{l}x_{2} \\ y_{2}\end{array}\right) \in \Lambda$, we have $x_{1}=x_{2}$ if and only if $y_{1}=y_{2}$. In fact,

$$
\begin{aligned}
0 & =\left\langle\tilde{J}\left(\begin{array}{l}
x_{1}-x_{2} \\
y_{1}-y_{2}
\end{array}\right),\left(\begin{array}{l}
x_{1}-x_{2} \\
y_{1}-y_{2}
\end{array}\right)\right\rangle \\
& =-\sqrt{-1}\left(\left(x_{1}-x_{2}\right)^{*}\left(x_{1}-x_{2}\right)-\left(y_{1}-y_{2}\right)^{*}\left(y_{1}-y_{2}\right)\right) .
\end{aligned}
$$


Then, we define a map $U: \mathbb{C}^{n_{1}} \rightarrow \mathbb{C}^{n_{2}}$ by $U(x)=y$ for for any vector $\left(\begin{array}{l}x \\ y\end{array}\right) \in \Lambda$. We claim that the domain and range are $\mathbb{C}^{n_{1}}$ and $\mathbb{C}^{n_{2}}$, respectively. Actually, we assume that

$$
\begin{aligned}
& D=\left\{x \in \mathbb{C}^{n_{1}} \mid \exists y \in \mathbb{C}^{n_{2}} \text {, satisfying }\left(\begin{array}{l}
x \\
y
\end{array}\right) \in \Lambda\right\}, \\
& R=\left\{y \in \mathbb{C}^{n_{2}} \mid \exists x \in \mathbb{C}^{n_{1}} \text {, satisfying }\left(\begin{array}{l}
x \\
y
\end{array}\right) \in \Lambda\right\} .
\end{aligned}
$$

Take $x \in D^{\perp}$ and then, from the definition of $D$, it follows $\left(\begin{array}{l}x \\ 0\end{array}\right) \in \Lambda^{\tilde{\omega}}=\Lambda$ by a simple computation as above. Thus we have $x=0$, i.e., $D^{\perp}=\{0\}$ and similarly $R^{\perp}=\{0\}$. So far, we have proved the claim. Furthermore, the map U is bijective and $n_{1}=n_{2}$.

Thus, assume that $\Lambda$ is represented as $\left\{\left(\begin{array}{c}x \\ U x\end{array}\right) \mid x \in \mathbb{C}^{n}\right\}$, where $U \in \mathcal{L}\left(\mathbb{C}^{n}\right)$ is an invertible matrix. Then, for any $x, y \in \mathbb{C}^{n}$, it follows

$$
\begin{aligned}
0 & =\left\langle\tilde{J}\left(\begin{array}{c}
x \\
U x
\end{array}\right),\left(\begin{array}{c}
y \\
U y
\end{array}\right)\right\rangle \\
& =\langle\sqrt{-1} x, y\rangle+\langle-\sqrt{-1} U x, U y\rangle \\
& =-\sqrt{-1} x^{*}\left(I-U^{*} U\right) y .
\end{aligned}
$$

By the arbitrariness of $x$ and $y$, we get $U^{*} U=I$.

Remark 2.10. Here there is a natural question: how can we generalize these concepts and ideas to manifold to obtain a meaningful complex symplectic geometry?

Henceforth, we assume that $n_{1}=n_{2}=n$ and denote the corresponding unitary matrix of a $\tilde{J}$-Lagrangian subspace $\Lambda$ by $\tilde{U}_{\Lambda}$. Due to this property, we can introduce a new Maslov index for a pair of $\tilde{J}$-Lagrangian paths.

Definition 2.11. For a pair of continuous $\tilde{J}$-Lagrangian paths $\Lambda_{1}(t), \Lambda_{2}(t) \in$ $C([0,1], \operatorname{Lag}(\tilde{J}))$, define a Maslov index by

$$
\operatorname{Mas}\left\{\Lambda_{1}(t), \Lambda_{2}(t)\right\}:=\operatorname{sf}\left\{\tilde{U}_{\Lambda_{1}(t)} \tilde{U}_{\Lambda_{2}(t)}^{-1}, t \in[0,1]\right\},
$$


where $\tilde{U}_{\Lambda_{j}(t)}, j=1,2$, is the corresponding unitary matrix of $\Lambda_{j}(t)$ obtained in Lemma 2.9.

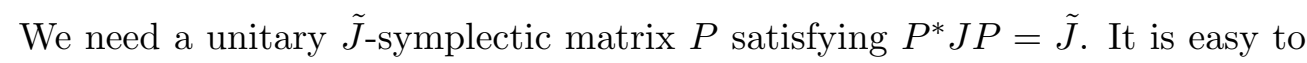
check that

$$
P=\frac{\sqrt{2}}{2}\left(\begin{array}{cc}
I & -\sqrt{-1} I \\
-\sqrt{-1} I & I
\end{array}\right)
$$

is suitable. Unless mentioned, we denote this matrix by $P$ henceforth.

For convenience, we introduce a new complex symplectic space $\left(\mathbb{C}^{2 n}, \tilde{\omega}_{\mathbb{C}}\right)$ with complex symplectic form $\tilde{\omega}_{\mathbb{C}}$ defined as,

$$
\tilde{\omega}_{\mathbb{C}}(u, v)=\langle J u, v\rangle_{\mathbb{C}}, \forall u, v \in \mathbb{C}^{2 n} .
$$

The set of all corresponding complex Lagrangian subspace is denoted by $\operatorname{Lag}(J)$. Moreover, a complex matrix $M \in \mathcal{L}\left(\mathbb{C}^{2 n}\right)$ is called $J$-symplectic, if

$$
M^{*} J M=J
$$

where $J$ is the standard (real) symplectic matrix defined as in Section 0 and all the $2 n \times 2 n J$-symplectic matrices form a subgroup of $\mathcal{L}\left(\mathbb{C}^{2 n}\right)$ denoted by $S p(J)$ and called the $J$-symplectic group.

Lemma 2.12. (a) If a complex Lagrangian subspace $\Lambda \in \operatorname{Lag}(J)$, then $P^{*} \Lambda \in$ $\operatorname{Lag}(\tilde{J})$; And conversely, if a complex Lagrangian subspace $\tilde{\Lambda} \in \operatorname{Lag}(\tilde{J})$, then $P \tilde{\Lambda} \in \operatorname{Lag}(J)$.

(b) If $M \in S p(J)$, then $\tilde{M}=P^{*} M P \in S p(\tilde{J}) ;$ And conversely, if $\tilde{M} \in S p(\tilde{J})$, then $M=P \tilde{M} P^{*} \in S p(J)$

Proof. (a) There are two approaches to this claim. One is the direct use of the definition:

$$
\tilde{\omega}\left(P^{*} \Lambda, P^{*} \Lambda\right)=\left\langle\tilde{J} P^{*} \Lambda, P^{*} \Lambda\right\rangle=\left\langle P \tilde{J} P^{*} \Lambda, \Lambda\right\rangle=\langle J \Lambda, \Lambda\rangle=0
$$

and in addition $\operatorname{dim} P^{*} \Lambda=n$. The other is to check that $\Lambda$ can be represented as $\operatorname{Gr}(U)$, where $U$ is an invertible matrix with $U^{*}=U$ and by a simple computation,

$$
P^{*} \Lambda=\operatorname{Gr}\left((U+\sqrt{-1} I)(\sqrt{-1} U+I)^{-1}\right) .
$$

By the result $U^{*}=U$, we have

$$
\left((U+\sqrt{-1} I)(\sqrt{-1} U+I)^{-1}\right)^{*}\left((U+\sqrt{-1} I)(\sqrt{-1} U+I)^{-1}\right)=I .
$$


So the criterion Lemma 2.9 implies $P^{*} \Lambda \in \operatorname{Lag}(\tilde{J})$. The converse can also be obtained by these two approaches.

(b) It can be easily obtained by a simple computation from the definition.

From this Lemma, it is obvious that $\operatorname{Lag}(J)$ and $\operatorname{Lag}(\tilde{J}), S p(J)$ and $S p(\tilde{J})$ are isomorphic, respectively. We will follow the notations in this Lemma henceforth. For example, $\tilde{\Lambda}$ and $\tilde{M}$ denote $P^{*} \Lambda$ and $P^{*} M P$, respectively, etc. It is obvious that

$$
\tilde{\Lambda}_{0}=\left\{\left(\begin{array}{c}
x \\
-\sqrt{-1} x
\end{array}\right) \mid x \in \mathbb{C}^{n}\right\}
$$

where $\Lambda_{0}=\left\{\left(\begin{array}{l}0 \\ x\end{array}\right) \mid x \in \mathbb{C}^{n}\right\}$. Moreover, $\operatorname{Sp}(2 n) \subset S p(J)$.

Proposition 2.13. (i) If $\Lambda \in \operatorname{Lag}(\tilde{J})$ and $\Psi \in \operatorname{Sp}(\tilde{J})$, then $\Psi \Lambda \in \operatorname{Lag}(\tilde{J})$.

(ii) For any two $\tilde{J}$-Lagrangian subspaces $\Lambda, \Lambda^{\prime} \in \operatorname{Lag}(\tilde{J})$ there exists a unitary $\tilde{J}$-symplectic matrix $\Psi \in S p(\tilde{J}) \cap \mathbb{U}(2 n)$ such that $\Lambda^{\prime}=\Psi \Lambda . \quad \Psi$ is uniquely determined by $\Lambda^{\prime}$ up to a unitary matrix $C \in \mathbb{U}(n)$, i.e., for any other choice of $\Psi^{\prime}$ satisfying the conditions above, there exists a unitary matrix $C \in \mathbb{U}(n)$ such that

$$
\Psi^{\prime}=\Psi\left(\begin{array}{ll}
C & 0 \\
0 & C
\end{array}\right)
$$

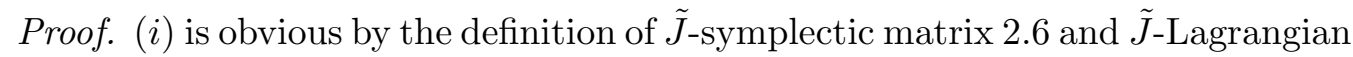
subspace.

(ii) By Lemma 2.9, $\Lambda^{\prime}$ can be written as $\left\{\left(\begin{array}{c}x \\ U^{\prime} x\end{array}\right) \mid x \in \mathbb{C}^{n}\right\}$ and thus $\Psi$ can be chosen as $\left(\begin{array}{lc}I & 0 \\ 0 & \sqrt{-1} U^{\prime}\end{array}\right)$ for $\Lambda=\tilde{\Lambda}_{0}$. Then Lemma 2.7 (b) proves the lemma.

Denote by

$$
\mathcal{P}(\tilde{J})=\left\{\gamma \in C([0,1], S p(\tilde{J})) \mid \gamma(0)=I_{2 n}\right\}
$$

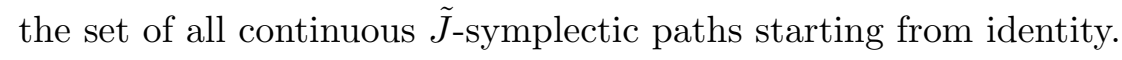

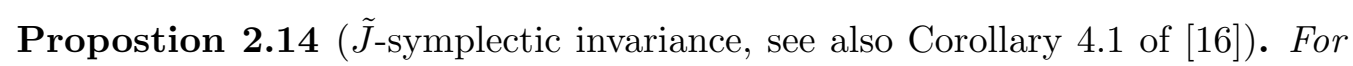
any $\gamma(t) \in \mathcal{P}(\tilde{J})$ and $\Lambda(t), \Lambda^{\prime}(t) \in C([0,1], \operatorname{Lag}(\tilde{J}))$, there holds

$$
\operatorname{Mas}\left\{\gamma(t) \Lambda^{\prime}(t), \gamma(t) \Lambda(t)\right\}=\operatorname{Mas}\left\{\Lambda^{\prime}(t), \Lambda(t)\right\} .
$$


Proof. Firstly, we claim that it suffices to prove

$$
\operatorname{Mas}\left\{\gamma(1) \Lambda^{\prime}(t), \gamma(1) \Lambda(t)\right\}=\operatorname{Mas}\left\{\Lambda^{\prime}(t), \Lambda(t)\right\} .
$$

In fact, by Definition 2.11,

$$
\operatorname{Mas}\left\{\gamma(t) \Lambda^{\prime}(t), \gamma(t) \Lambda(t)\right\}=-s f\left\{\tilde{U}_{\gamma(t) \Lambda^{\prime}(t)} \tilde{U}_{\gamma(t) \Lambda(t)}^{-1}, t \in[0,1]\right\},
$$

i.e., we can prove

$$
\operatorname{Mas}\left\{\gamma(t) \Lambda^{\prime}(t), \gamma(t) \Lambda(t)\right\}=\operatorname{Mas}\left\{\gamma(1) \Lambda^{\prime}(t), \gamma(1) \Lambda(t)\right\} .
$$

Here $M \triangleq \gamma(1)$. Supposing that the eigenvalues of $\tilde{U}_{\Lambda^{\prime}(t)} \tilde{U}_{\Lambda(t)}^{-1}$ are $e^{\sqrt{-1} \theta_{j}(t)}$ and those of $\tilde{U}_{M \Lambda^{\prime}(t)} \tilde{U}_{M \Lambda(t)}^{-1}$ are $e^{\sqrt{-1} \theta_{M_{j}}(t)}, j=1,2, \cdots, n$, we have

$$
\begin{aligned}
& -\sum_{j=1}^{n}\left(\left[\frac{\theta_{M j}(1)}{2 \pi}\right]-\left[\frac{\theta_{j}(0)}{2 \pi}\right]\right) \\
= & -\sum_{j=1}^{n}\left(\left[\frac{\theta_{M j}(1)}{2 \pi}\right]-\left[\frac{\theta_{M j}(0)}{2 \pi}\right]+\left[\frac{\theta_{M j}(0)}{2 \pi}\right]-\left[\frac{\theta_{j}(0)}{2 \pi}\right]\right) \\
= & -\sum_{j=1}^{n}\left(\left[\frac{\theta_{M j}(1)}{2 \pi}\right]-\left[\frac{\theta_{M j}(0)}{2 \pi}\right]\right) \\
= & \operatorname{Mas}\left\{M \Lambda^{\prime}(t), M \Lambda(t)\right\},
\end{aligned}
$$

where the last second equality follows from

$$
\operatorname{sf}\left\{\tilde{U}_{\gamma(t) \Lambda^{\prime}(0)} \tilde{U}_{\gamma(t) \Lambda(0)}^{-1}, t \in[0,1]\right\}=0 .
$$

Actually, by the fact

$$
\gamma(t)\left(\Lambda^{\prime}(0) \cap \Lambda(0)\right) \cong M\left(\Lambda^{\prime}(0) \cap \Lambda(0)\right) \cong\left(\Lambda^{\prime}(0) \cap \Lambda(0)\right),
$$

it follows that $\operatorname{dim} \gamma(t)\left(\Lambda^{\prime}(0) \cap \Lambda(0)\right)=$ constant, i.e.,

$$
\operatorname{dim} \operatorname{ker}\left(I-\tilde{U}_{\gamma(t) \Lambda^{\prime}(0)} \tilde{U}_{\gamma(t) \Lambda(0)}^{-1}\right)=\text { constant }
$$

Thus by the Property Zero of spectral flow (cf. Proposition 2.5(d)), we can obtain this claim.

Next, we prove

$$
\operatorname{Mas}\left\{M \Lambda^{\prime}(t), M \Lambda(t)\right\}=\operatorname{Mas}\left\{\Lambda^{\prime}(t), \Lambda(t)\right\} .
$$


Actually,

$$
\begin{aligned}
& \operatorname{Mas}\left\{M \Lambda^{\prime}(t), M \Lambda(t)\right\}-\operatorname{Mas}\left\{\gamma(0) \Lambda^{\prime}(t), \gamma(0) \Lambda(t)\right\} \\
= & \operatorname{Mas}\left\{\gamma(s) \Lambda^{\prime}(1), \gamma(s) \Lambda(1)\right\}-\operatorname{Mas}\left\{\gamma(s) \Lambda^{\prime}(0), \gamma(s) \Lambda(0)\right\} \\
= & 0
\end{aligned}
$$

where the first equality follows from the Property Homotopy invariance of spectral flow (cf. Proposition 2.5(c)) and the second equality follows from

$$
\operatorname{Mas}\left\{\gamma(s) \Lambda^{\prime}(1), \gamma(s) \Lambda(1)\right\}=\operatorname{Mas}\left\{\gamma(s) \Lambda^{\prime}(0), \gamma(s) \Lambda(0)\right\}=0
$$

by the same reasoning of (2.6).

2.3. An explicit formula about Lagrangian-index and spectral flow. Let us introduce a new index by the Maslov index in Definition 2.11 with respect

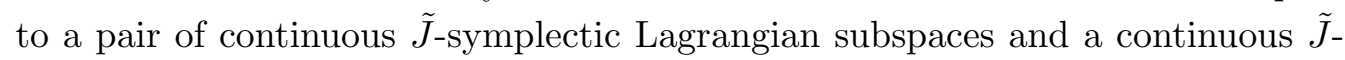
symplectic path in $S p(\tilde{J})$.

Definition 2.15. For any $\Lambda(t), \Lambda^{\prime}(t) \in C([0,1], \operatorname{Lag}(\tilde{J}))$ and $\tilde{\gamma} \in C([0,1], S p(\tilde{J}))$, we define $\left(\Lambda, \Lambda^{\prime}\right)$-index of $\tilde{\gamma}$ by

$$
i_{\Lambda, \Lambda^{\prime}}(\tilde{\gamma})=\operatorname{Mas}\left\{\tilde{\gamma}(t) \Lambda(t), \Lambda^{\prime}(t)\right\} .
$$

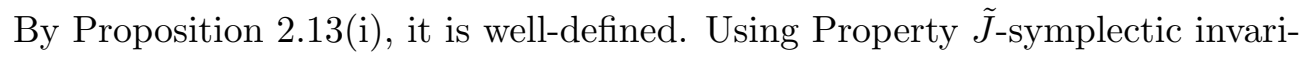
ance of the Maslov index (cf. Proposition 2.14), we can reduce the $\left(\Lambda, \Lambda^{\prime}\right)$-index to the special case $\left(\tilde{\Lambda}_{0}, \tilde{\Lambda}_{0}\right)$-index. In fact,

$$
\begin{aligned}
i_{\Lambda, \Lambda^{\prime}}(\tilde{\gamma}) & =\operatorname{Mas}\left\{\tilde{\gamma}(t) \Lambda(t), \Lambda^{\prime}(t)\right\} \\
& =\operatorname{Mas}\left\{\tilde{\gamma}(t) \Psi(t) \tilde{\Lambda}_{0}, \Psi^{\prime}(t) \tilde{\Lambda}_{0}\right\} \\
& =\operatorname{Mas}\left\{\Psi^{\prime}(t)^{-1} \tilde{\gamma}(t) \Psi(t) \tilde{\Lambda}_{0}, \tilde{\Lambda}_{0}\right\} \\
& =\operatorname{Mas}\left\{\bar{\gamma}(t) \tilde{\Lambda}_{0}, \tilde{\Lambda}_{0}\right\}
\end{aligned}
$$

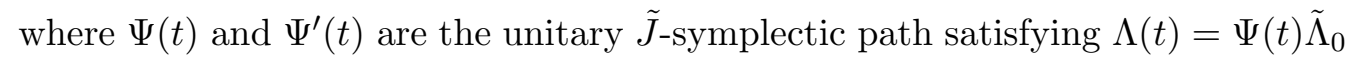
and $\Lambda^{\prime}(t)=\Psi^{\prime}(t) \tilde{\Lambda}_{0}$ (cf. Proposition 2.13(ii)), and $\bar{\gamma}(t) \triangleq \Psi^{\prime}(t)^{-1} \tilde{\gamma}(t) \Psi(t)$. And

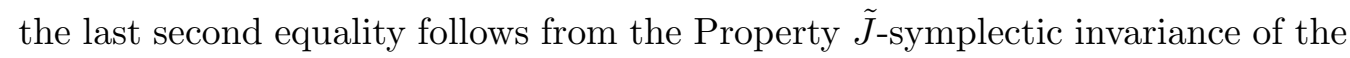
Maslov index. 
Next, we show that $\operatorname{Mas}\left\{\bar{\gamma}(t) \tilde{\Lambda}_{0}, \tilde{\Lambda}_{0}\right\}$ is independent of the choice of $\Psi(t)$ and $\Psi^{\prime}(t)$. In fact, for another choice of $\Psi_{0}(t)$ and $\Psi_{0}^{\prime}(t)$ satisfying

$$
\Psi_{0}(t)=\Psi(t)\left(\begin{array}{cc}
C(t) & 0 \\
0 & C(t)
\end{array}\right) \text { and } \Psi_{0}^{\prime}(t)=\Psi^{\prime}(t)\left(\begin{array}{cc}
C^{\prime}(t) & 0 \\
0 & C^{\prime}(t)
\end{array}\right),
$$

where $C(t), C^{\prime}(t)$ are unitary paths as in Proposition 2.13(ii) and

$$
\bar{\gamma}_{0}(t) \triangleq \Psi_{0}^{\prime}(t)^{-1} \tilde{\gamma}(t) \Psi_{0}(t)
$$

we have

$$
\begin{aligned}
\bar{\gamma}_{0}(t) \tilde{\Lambda}_{0} & =\left(\begin{array}{cc}
C^{\prime}(t)^{-1} & 0 \\
0 & C^{\prime}(t)^{-1}
\end{array}\right) \Psi^{\prime}(t)^{-1} \tilde{\gamma}(t) \Psi(t)\left(\begin{array}{cc}
C(t) & 0 \\
0 & C(t)
\end{array}\right) \tilde{\Lambda}_{0}(t) \\
& =\left(\begin{array}{cc}
C^{\prime}(t)^{-1} & 0 \\
0 & C^{\prime}(t)^{-1}
\end{array}\right) \operatorname{Gr}\left(\tilde{U}_{\bar{\gamma}(t) \tilde{\Lambda}_{0}(t)}\right) \\
& =\left\{\left(\begin{array}{c}
x \\
C^{\prime}(t)^{-1} \tilde{U}_{\bar{\gamma}(t) \tilde{\Lambda}_{0}(t)} C^{\prime}(t) x
\end{array}\right) \mid x \in \mathbb{C}^{n}\right\} .
\end{aligned}
$$

Since $C^{\prime}(t)^{-1} \tilde{U}_{\bar{\gamma}(t) \tilde{\Lambda}_{0}(t)} C^{\prime}(t)$ does not change the eigenvalues of $\tilde{U}_{\bar{\gamma}(t) \tilde{\Lambda}_{0}(t)}$, we obtain the independence by the definition of Maslov index.

In the end of this section, we give the main result of this paper.

Theorem 2.16. For any symplectic path $\gamma \in \mathcal{P}(2 n)$, there holds

$$
i_{L_{0}}(\gamma)=i_{\tilde{\Lambda}_{0}, \tilde{\Lambda}_{0}}(\tilde{\gamma})-n
$$

Recall that $L_{0}=\{0\} \oplus \mathbb{R}^{n}$ and $\tilde{\Lambda}_{0}$ is given by (2.5). $\tilde{\gamma}$ denotes $P^{*} \gamma P$ where $P$ is given by (2.4).

Proof. Write $\gamma(t) \triangleq\left(\begin{array}{c}S(t) V(t) \\ T(t) U(t)\end{array}\right)$ as in (1.1). Suppose $\lambda_{j}(t)=e^{2 \sqrt{-1} \theta_{j}(t)}$ are the eigenvalues of $Q_{\gamma}(t)$ for $j=1,2, \cdots, n$, where

$$
Q_{\gamma}(t)=[U(t)-\sqrt{-1} V(t)][U(t)+\sqrt{-1} V(t)]^{-1}
$$

as in (1.2). Using the result and notations in Theorem 1.3, we have

$$
\begin{aligned}
i_{L_{0}}(\gamma) & =\sum_{j=1}^{n} E\left(\frac{\theta_{j}(1)-\theta_{j}(0)}{\pi}\right) \\
& =-\sum_{j=1}^{n}\left[-\left(\frac{\theta_{j}(1)-\theta_{j}(0)}{\pi}\right)\right]-n
\end{aligned}
$$




$$
\begin{aligned}
& =-\sum_{j=1}^{n}\left(\left[-\frac{\theta_{j}(1)}{\pi}\right]-\left[-\frac{\theta_{j}(0)}{\pi}\right]\right)-n \\
& =-\operatorname{sf}\left\{\left((U(t)-\sqrt{-1} V(t))(U(t)+\sqrt{-1} V(t))^{-1}\right)^{-1}, t \in[0,1]\right\}-n
\end{aligned}
$$

$=\operatorname{Mas}\left\{\left\{\left(\begin{array}{c}x \\ -\sqrt{-1}(U(t)+\sqrt{-1} V(t))(U(t)-\sqrt{-1} V(t))^{-1} x\end{array}\right) \mid x \in \mathbb{C}^{n}\right\}, \tilde{\Lambda}_{0}\right\}-n$

$=\operatorname{Mas}\left\{\left(\begin{array}{cc}I & \sqrt{-1} I \\ \sqrt{-1} I & I\end{array}\right)\left(\begin{array}{l}S(t) V(t) \\ T(t) U(t)\end{array}\right)\left\{\left(\begin{array}{l}0 \\ x\end{array}\right) \mid x \in \mathbb{C}^{n}\right\}, \tilde{\Lambda}_{0}\right\}-n$

$=\operatorname{Mas}\left\{P^{*} \gamma P P^{*} \Lambda_{0}, \tilde{\Lambda}_{0}\right\}-n$

$=\operatorname{Mas}\left\{P^{*} \gamma P \tilde{\Lambda}_{0}, \tilde{\Lambda}_{0}\right\}-n$

$=\operatorname{Mas}\left\{\tilde{\gamma} \tilde{\Lambda}_{0}, \tilde{\Lambda}_{0}\right\}-n$,

where the first and second equalities follow from Theorem 1.3 and the formula (2.2) respectively.

Remark 2.17. The critical third equality can also be obtained by the first equality, Proposition 2.5 (b)(Reversal), $\gamma(0)=I$ and the fact that the total algebraic multiplicities of eigenvalues is $n$. In fact,

$$
\begin{aligned}
i_{L_{0}}(\gamma) & =\sum_{j=1}^{n} E\left(\frac{\theta_{j}(1)-\theta_{j}(0)}{\pi}\right) \\
& =\sum_{j=1}^{n}\left(\left(\left[\frac{\theta_{j}(1)}{\pi}\right]-\delta\left(\frac{\theta_{j}(1)}{\pi}\right)\right)-\left[\frac{\theta_{j}(0)}{\pi}\right]\right) \\
& =-\sum_{j=1}^{n}\left(\left[-\frac{\theta_{j}(1)}{\pi}\right]-\left[-\frac{\theta_{j}(0)}{\pi}\right]\right)+\sum_{j=1}^{n}\left(\delta\left(\frac{\theta_{j}(1)}{\pi}\right)-1\right)-\sum_{j=1}^{n} \delta\left(\frac{\theta_{j}(1)}{\pi}\right) \\
& =-\sum_{j=1}^{n}\left(\left[-\frac{\theta_{j}(1)}{\pi}\right]-\left[-\frac{\theta_{j}(0)}{\pi}\right]\right)-n .
\end{aligned}
$$

\section{A NEW INDEX WITH ARBITRARY BOUNDARY CONDITIONS}

We generalize our main theorem to arbitrary Lagrangian boundary conditions by the methods developed in the previous section and some results from Lie theory. 
3.1. The index theory with arbitrary boundary conditions. On the basis of the Lagrangian-index mentioned above, we develop a new index which is suitable to study a more general case, that is, to study the following linear Hamiltonian system with arbitrary Lagrangian boundary conditions

$$
\left\{\begin{array}{l}
\dot{x}(t)=J B(t) x(t) \\
x(0) \in L_{0} \\
x(1) \in L_{1}
\end{array}\right.
$$

where $B(t), t \in[0,1]$, is still a family of symmetric matrix functions, and $L_{1} \in$ $\operatorname{Lag}(n)$ is an arbitrary Lagrangian subspace of $\mathbb{R}^{2 n}$.

Suppose that $\gamma(t)$ is the fundamental solution of the linear Hamiltonian system $\dot{x}(t)=J B(t) x(t)$, that is, $\gamma(0)=I_{2 n}$ the identity matrix, and $\dot{\gamma}(t)=J B(t) \gamma(t)$, then $\gamma \in C([0,1], S p(2 n))$ is a symplectic path with $\gamma(0)=I_{2 n}$ and write it in the form $\left(\begin{array}{l}S(t) V(t) \\ T(t) U(t)\end{array}\right)$, where $S(t), T(t), V(t)$ and $U(t)$ are all $n \times n$ matrices. It is easy to see that the $n$ vectors coming from the column of the matrix $\left(\begin{array}{l}V(t) \\ U(t)\end{array}\right)$ are linear independent and they span a Lagrangian subspace of $\left(\mathbb{R}^{2 n}, \omega_{0}\right)$.

For the linear Lagrangian subspaces $L_{0}$ and $L_{1}$, there exists an orthogonal symplectic matrix $P$ such that $P L_{0}=L_{1}$ and $P$ has the form $P=\left(\begin{array}{c}A-B \\ B\end{array}\right)$ with $A \pm \sqrt{-1} B \in \mathbb{U}(n)$, the unitary matrix. So $P^{-1}=P^{T}=\left(\begin{array}{cc}A^{T} & B^{T} \\ -B^{T} & A^{T}\end{array}\right)$. Here $P$ is uniquely determined by $L_{0}$ and $L_{1}$ up to an orthogonal matrix $C \in O(n)$, where $O(n)$ denotes the orthogonal $n \times n$ matrix group. (cf. Lemma 2.31 in [13].) Moreover, the symplectic group is a Lie group and its Lie algebra is

$$
s p(2 n)=\left\{M \in \mathcal{L}\left(R^{2 n}, R^{2 n}\right) \mid J M+M^{T} J=0\right\} .
$$

It is easy to see that $J e^{t M}=e^{-t M^{T}} J$, where $M^{T}$ denotes the transpose of the matrix $M$. Hence for any symplectic matrix $N \in S p(2 n)$, we have

$$
\left(e^{-t M} N\right)^{T} J\left(e^{-t M} N\right)=N^{T} e^{-t M^{T}} J e^{-t M} N=N^{T} J N=J,
$$

that is, $e^{-t M} N \in S p(2 n)$. For any $M \in S p(2 n)$, it is well-known that the one parameter curve $\exp (t M)=e^{t M}$ in $S p(2 n)$ is a Lie subgroup of $S p(2 n)$, and 
$S p(2 n)=\exp (s p(2 n))$, which means the exponential map

$$
\exp : s p(2 n) \longrightarrow S p(2 n)
$$

is surjective (not injective). So for the above $P \in S p(2 n) \cap O(2 n)$, there is a matrix $M \in \operatorname{sp}(2 n)$ such that $P=e^{M}$ and $e^{t M} \in S p(2 n) \cap O(2 n)$. Furthermore, $M$ is anti-symmetric, that is, $M^{T}+M=0$. So by $J M+M^{T} J=0$, we have $J M=M J$. Thus we have a Lagrangian path $\lambda:[0,1] \longrightarrow \operatorname{Lag}(n)$ with $\lambda(t)=e^{t M} L_{0}$. We write the orthogonal symplectic path

$$
\gamma_{0}(t)=e^{t M}=\left(\begin{array}{ll}
A(t) & B(t) \\
-B(t) & A(t)
\end{array}\right) \text { with } A(t) \pm \sqrt{-1} B(t) \in \mathbb{U}(n) .
$$

The above Lagrangian path determines a unique unitary matrix path

$$
\mathfrak{R}_{0}(t)=[A(t)-\sqrt{-1} B(t)][A(t)+\sqrt{-1} B(t)]^{-1} .
$$

But the matrix $M$ is not unique since $\check{M}:=M+2 k \pi J \in \operatorname{sp}(2 n)$ also satisfies $e^{\check{M}}=P$ and we get another symplectic path $\gamma_{k}(t)=e^{t(M+2 k \pi J)}$ with $\mathfrak{R}_{k}(t)=$ $e^{4 k \pi t} \mathfrak{R}_{0}(t)$.

Proposition 3.1. Let $\gamma_{0}(t)=e^{t M}$ and $\gamma_{k}(t)=e^{t(M+2 k \pi J)}$ be the two symplectic paths with the same end points. Then we have

$$
i_{L_{0}}\left(\gamma_{k}\right)=2 n k+i_{L_{0}}\left(\gamma_{0}\right)
$$

Proof. Let us follow the notions as in paper [6]. Then

$$
\gamma_{k}(t)=\left(\begin{array}{c}
S_{k}(t) V_{k}(t) \\
T_{k}(t) U_{k}(t)
\end{array}\right) \text { and } \mathcal{Q}_{k}(t)=\left[U_{k}(t)-\sqrt{-1} V_{k}(t)\right]\left[U_{k}(t)+\sqrt{-1} V_{k}(t)\right]^{-1} .
$$

By the fact $\gamma_{k}(t)=e^{2 k \pi J t} \gamma_{0}(t)$, we have

$$
\mathcal{Q}_{k}(t)=e^{4 \sqrt{-1} k \pi t} \mathcal{Q}_{0}(t) .
$$

By Theorem 3.3 of [6], we get the result (3.2).

From Proposition 3.2, since $i_{L_{0}}\left(I_{2 n}\right)=-n$, we choose a unique matrix $M$ in the set

$$
\Theta=\left\{M \in s p(2 n) \mid e^{M}=P, J M=M J \text { and } i_{L_{0}}\left(e^{-t M}\right) \in[-n, n)\right\} .
$$

In the rest, we always fix such a matrix $M \in \Theta$. 
Definition 3.2. For any symplectic path $\gamma(t)=\left(\begin{array}{c}S(t) V(t) \\ T(t) U(t)\end{array}\right)$, define the $\left(L_{0}, L_{1}\right)$ nullity to be

$$
\nu_{L_{0}}^{L_{1}}(\gamma) \equiv \operatorname{dim} \operatorname{ker}\left(A^{T} V(1)+B^{T} U(1)\right)
$$

In fact, $\nu_{L_{0}}^{L_{1}}(\gamma)$ is just the dimension of the solution space of the problem (3.1). Let $x=(p, q) \in \mathbb{R}^{n} \times \mathbb{R}^{n}$ be a solution of (3.1). Then it satisfies $x(t)=\gamma(t) x(0)$. Suppose $(0, v) \in\{0\} \times \mathbb{R}^{n}$ and $P\left(0, v^{\prime}\right)=(p, q)$ for some $\left(0, v^{\prime}\right) \in\{0\} \times \mathbb{R}^{n}$. Hence by the equality

$$
\left(\begin{array}{l}
p \\
q
\end{array}\right)=\left(\begin{array}{l}
A-B \\
B A
\end{array}\right)\left(\begin{array}{l}
0 \\
v^{\prime}
\end{array}\right)=\left(\begin{array}{l}
S(1) V(1) \\
T(1) U(1)
\end{array}\right)\left(\begin{array}{l}
0 \\
v
\end{array}\right),
$$

we see that $\left(A^{T} V(1)+B^{T} U(1)\right) v=0$.

Remark 3.3. The definition is well-defined. Since for another $P^{\prime}$ satisfies the condition we have

$$
P^{\prime}=P\left(\begin{array}{ll}
C & 0 \\
0 & C
\end{array}\right)=\left(\begin{array}{ll}
A-B \\
B & A
\end{array}\right)\left(\begin{array}{ll}
C & 0 \\
0 & C
\end{array}\right)=\left(\begin{array}{l}
A C-B C \\
B C A C
\end{array}\right),
$$

where $C \in O(n)$, then

$$
\begin{aligned}
\nu_{L_{0}}^{L_{1}}(\gamma) & =\operatorname{dim} \operatorname{ker}\left((A C)^{T} V(1)+(B C)^{T} U(1)\right) \\
& =\operatorname{dim} \operatorname{ker}\left(A^{T} V(1)+B^{T} U(1)\right) .
\end{aligned}
$$

Definition 3.4. For the symplectic path $\gamma \in \mathcal{P}(2 n)$, we use the $L_{0}$-index to define the $\left(L_{0}, L_{1}\right)$-index of $\gamma$ by

$$
i_{L_{0}}^{L_{1}}(\gamma) \equiv i_{L_{0}}\left(e^{-t M} \gamma(t)\right)
$$

Remark 3.5. The index $i_{L_{0}}^{L_{1}}(\gamma)$ is well-defined, that is, it is independent of the choice of the matrixes $M$ and $P$. We just need consider how to ensure the independence of the choice of the orthogonal symplectic matrix P. For the other choice $P^{\prime}$, there is an orthogonal matrix $C \in O(n)$ such that $P^{\prime}=P\left(\begin{array}{ll}C & 0 \\ 0 & C\end{array}\right)$. We divide our work into two steps.

Step 1. If $\operatorname{det} C=1$, then

$$
\left(\begin{array}{ll}
C & 0 \\
0 & C
\end{array}\right)=\left(\begin{array}{cc}
e^{S} & 0 \\
0 & e^{S}
\end{array}\right)=\exp \left(\begin{array}{ll}
S & 0 \\
0 & S
\end{array}\right),
$$


and

$$
P^{\prime}=e^{M} \exp \left(\begin{array}{ll}
S & 0 \\
0 & S
\end{array}\right)=\exp \left(M+\left(\begin{array}{ll}
S & 0 \\
0 & S
\end{array}\right)\right) \text {. }
$$

The symplectic path $\exp \left(-t M+\left(\begin{array}{cc}-t S & 0 \\ 0 & -t S\end{array}\right)\right) \gamma(t)$ is $L_{0}$-homotopic to $e^{-t M} \gamma(t)$ by the homotopy

$$
\exp \left(-t M+\left(\begin{array}{cc}
-s t S & 0 \\
0 & -s t S
\end{array}\right)\right) \gamma(t), \quad(s, t) \in[0,1] \times[0,1] .
$$

(See Definition 3.9 about ' $L_{0}$-homotopic'.) Thus in this case $i_{L_{0}}^{L_{1}}(\gamma)$ is independent of the choice of $C$.

Step 2. If $\operatorname{det} C=-1$, then

$$
P^{\prime}=P\left(\begin{array}{cccc}
-1 & 0 & 0 & 0 \\
0 & I_{n-1} & 0 & 0 \\
0 & 0 & -1 & 0 \\
0 & 0 & 0 & I_{n-1}
\end{array}\right)\left(\begin{array}{cc}
C^{\prime} & 0 \\
0 & C^{\prime}
\end{array}\right) \text {, with } \operatorname{det} C^{\prime}=1
$$

By Step 1, we only need to suppose that

$$
P^{\prime}=P\left(\begin{array}{cccc}
-1 & 0 & 0 & 0 \\
0 & I_{n-1} & 0 & 0 \\
0 & 0 & -1 & 0 \\
0 & 0 & 0 & I_{n-1}
\end{array}\right)=e^{M} e^{\pi J^{\prime}}=e^{M+\pi J^{\prime}} \text { with } J^{\prime}=J_{2} \diamond I_{2 n-2}
$$

In this case, the symplectic paths $e^{-t \pi J^{\prime}} e^{-t M} \gamma(t)$ and $e^{-t M} \gamma(t)$ satisfy

$$
i_{L_{0}}\left(e^{-t M} \gamma(t)\right)=i_{L_{0}}\left(e^{-t \pi J^{\prime}} e^{-t M} \gamma(t)\right)+1 .
$$

For the proof of this result, one can use the similar arguments as in the proof of Proposition 3.2. By this, from now on, we fix an orthogonal symplectic matrix $P$ such that $P: L_{0} \rightarrow L_{1}$ be orientation preserving, i.e., just the case in the Step 1.

Generally, we consider the following Hamiltonian system

$$
\left\{\begin{array}{l}
\dot{x}(t)=J B(t) x(t) \\
x(0) \in L \\
x(1) \in L_{1}
\end{array}\right.
$$


where $L$ and $L_{1}$ are two arbitrary Lagrangian subspaces in $\mathbb{R}^{2 n}$. We also can find an orthogonal symplectic matrix $O$ satisfying $O L_{0}=L$ and thus define a new symplectic path $\gamma_{O}(t)=O^{-1} \gamma(t) O$.

Definition 3.6. The $\left(L, L_{1}\right)$-nullity of any symplectic path $\gamma \in \mathcal{P}(2 n)$ is defined by

$$
\nu_{L}^{L_{1}}(\gamma)=\operatorname{dim} \operatorname{ker}\left(A_{1}^{T} V_{O}(1)+B_{1}^{T} U_{O}(1)\right),
$$

where $V_{O}(t)$ and $U_{O}(t)$ are defined in $\gamma_{O}(t)=\left(\begin{array}{c}S_{O}(t) V_{O}(t) \\ T_{O}(t) U_{O}(t)\end{array}\right)$ and $P_{1}=\left(\begin{array}{l}A_{1}-B_{1} \\ B_{1} A_{1}\end{array}\right)$ satisfies $P_{1} L_{0}=O^{-1} L_{1}$.

Remark 3.7. This definition is well-defined. Since if the other $O^{\prime}=O\left(\begin{array}{ll}C^{\prime} & 0 \\ 0 & C^{\prime}\end{array}\right)$ satisfies the above conditions, we have

$$
\begin{aligned}
\left(O^{\prime}\right)^{-1} \gamma(t) O^{\prime} & =\left(\begin{array}{ll}
\left(C^{\prime}\right)^{-1} & 0 \\
0 & \left(C^{\prime}\right)^{-1}
\end{array}\right) O^{-1} \gamma(t) O\left(\begin{array}{ll}
C^{\prime} & 0 \\
0 & C^{\prime}
\end{array}\right) \\
& =\left(\begin{array}{ll}
\left(C^{\prime}\right)^{-1} 0 \\
0 & \left(C^{\prime}\right)^{-1}
\end{array}\right)\left(\begin{array}{l}
S_{O}(t) V_{O}(t) \\
T_{O}(t) U_{O}(t)
\end{array}\right)\left(\begin{array}{ll}
C^{\prime} & 0 \\
0 & C^{\prime}
\end{array}\right) \\
& =\left(\begin{array}{l}
\left(C^{\prime}\right)^{-1} S_{O}(t) C^{\prime}\left(C^{\prime}\right)^{-1} V_{O}(t) C^{\prime} \\
\left(C^{\prime}\right)^{-1} T_{O}(t) C^{\prime}\left(C^{\prime}\right)^{-1} U_{O}(t) C^{\prime}
\end{array}\right) .
\end{aligned}
$$

Hence, we have

$$
\begin{aligned}
\nu_{L}^{L_{1}}(\gamma)=\operatorname{dim} \operatorname{ker}\left(\left(C^{\prime}\right)^{-1} A_{1}^{T} V_{O}(1) C^{\prime}+\left(C^{\prime}\right)^{-1} B_{1}^{T} U_{O}(1) C^{\prime}\right) & \\
& =\operatorname{dim} \operatorname{ker}\left(A_{1}^{T} V_{O}(1)+B_{1}^{T} U_{O}(1)\right)
\end{aligned}
$$

and thus the definition is independent of the choice of the matrix $O$.

Definition 3.8. The $\left(L, L_{1}\right)$-index is defined by

$$
i_{L}^{L_{1}}(\gamma)=i_{L_{0}}\left(e^{-t M_{1}} \gamma_{O}(t)\right)
$$

where $e^{M_{1}}=P_{1}$ and $i_{L_{0}}\left(e^{-t M_{1}}\right) \in[-n, n)$.

We claim that this definition is independent of the choice of the matrix $O \in$ $S p(2 n) \cap O(2 n)$. Since for another matrix $O^{\prime}=O\left(\begin{array}{ll}C^{\prime} & 0 \\ 0 & C^{\prime}\end{array}\right)$, we have

$$
\left(O^{\prime}\right)^{-1} \gamma(t) O^{\prime}=\left(\begin{array}{l}
\left(C^{\prime}\right)^{-1} S_{O}(t) C^{\prime}\left(C^{\prime}\right)^{-1} V_{O}(t) C^{\prime} \\
\left(C^{\prime}\right)^{-1} T_{O}(t) C^{\prime}\left(C^{\prime}\right)^{-1} U_{O}(t) C^{\prime}
\end{array}\right)
$$


Therefore

$$
\begin{aligned}
& \operatorname{det}\left[\left(C^{\prime}\right)^{-1} U_{O}(t) C^{\prime}-\sqrt{-1}\left(C^{\prime}\right)^{-1} V_{O}(t) C^{\prime}\right]\left[\left(C^{\prime}\right)^{-1} U_{O}(t) C^{\prime}+\sqrt{-1}\left(C^{\prime}\right)^{-1} V_{O}(t) C^{\prime}\right]^{-1} \\
= & \operatorname{det}\left[U_{O}(t)-\sqrt{-1} V_{O}(t)\right]\left[U_{O}(t)+\sqrt{-1} V_{O}(t)\right]^{-1} .
\end{aligned}
$$

3.2. Some properties of our new index. Our new index defined in Subsection 3.1 has many properties which are similar to the Lagrangian-index developed by C. Liu. For convenience, we first briefly list some results in the paper [6]. In the paper [8], the authors obtained many more results by using the methods of Galerkin approximation and the saddle point reduction. First we give some necessary definitions for the statement.

Definition 3.9. (i) For two symplectic paths $\gamma_{0}, \gamma_{1} \in \mathcal{P}(2 n)$, we say that they are $L_{0}$-homotopic and denoted by $\gamma_{0} \sim_{L_{0}} \gamma_{1}$, if there is a map $\delta:[0,1] \rightarrow \mathcal{P}(2 n)$ such that $\delta(j)=\gamma_{j}$ for $j=0,1$, and $\nu_{L_{0}}(\delta(s))$ is constant for $s \in[0,1]$.

(ii) For two symplectic matrices of the square block form

$$
M_{1}=\left(\begin{array}{ll}
A_{1} & B_{1} \\
C_{1} & D_{1}
\end{array}\right)_{2 i \times 2 i} \quad \text { and } \quad M_{2}=\left(\begin{array}{ll}
A_{2} & B_{2} \\
C_{2} & D_{2}
\end{array}\right)_{2 j \times 2 j},
$$

the symplectic direct sum of $M_{1}$ and $M_{2}$ is defined by

$$
M_{1} \diamond M_{2}=\left(\begin{array}{cccc}
A_{1} & 0 & B_{1} & 0 \\
0 & A_{2} & 0 & B_{2} \\
C_{1} & 0 & D_{1} & 0 \\
0 & C_{2} & 0 & D_{2}
\end{array}\right) .
$$

In his paper [6], C. Liu also proved the following important result.

Theorem $3.10([6])$. (i) If $\gamma_{0}, \gamma_{1} \in \mathcal{P}(2 n)_{L_{0}}^{*}$, then $i_{L_{0}}\left(\gamma_{0}\right)=i_{L_{0}}\left(\gamma_{1}\right)$ if and only if $\gamma_{0} \sim_{L_{0}} \gamma_{1}$.

(ii) For two symplectic paths $\gamma_{j} \in \mathcal{P}\left(2 n_{j}\right), j=1,2$, with $n_{1}+n_{2}=n$, there holds

$$
i_{L_{0}}\left(\gamma_{1} \diamond \gamma_{2}\right)=i_{L_{0}^{\prime}}\left(\gamma_{1}\right)+i_{L_{0}^{\prime \prime}}\left(\gamma_{2}\right),
$$

where $L_{0}^{\prime}=\{0\} \oplus \mathbb{R}^{n_{1}}$ and $L_{0}^{\prime \prime}=\{0\} \oplus \mathbb{R}^{n_{2}}$.

(iii) For two symplectic paths $\gamma_{j} \in \mathcal{P}\left(2 n_{j}\right), j=1,2$ with $\gamma_{1}(1)=\gamma_{2}(1)$, it holds $i_{L_{0}}\left(\gamma_{1}\right)=i_{L_{0}}\left(\gamma_{2}\right)$ if and only if $\gamma_{1} \sim_{L_{0}} \gamma_{2}$ with fixed endpoints. 
(iv) For two symplectic paths $\gamma_{j} \in \mathcal{P}\left(2 n_{j}\right), j=1,2$, if $\gamma_{1} \sim_{L_{0}} \gamma_{2}$, there holds

$$
i_{L_{0}}\left(\gamma_{1}\right)=i_{L_{0}}\left(\gamma_{2}\right), \nu_{L_{0}}\left(\gamma_{1}\right)=\nu_{L_{0}}\left(\gamma_{2}\right)
$$

(v) For any symplectic path $\gamma \in \mathcal{P}(2 n)$, there holds

$$
i_{L_{0}}(\gamma)=\sum_{j=1}^{n} E\left(\frac{\theta_{j}(1)-\theta_{j}(0)}{\pi}\right)
$$

where $E(a)=\max \{k \in \mathbb{Z} \mid k<a\}$.

Now we list some properties of our new index below. Since we use the Lagrangianindex to define the new index and the fact that $e^{-t M} \gamma(t)$ is the fundamental solution of $\dot{x}=J \tilde{B} x(t)$, where $\tilde{B}=J M+e^{-t M} B(t) e^{t M}$, these properties of the new index are very similar to those of the Lagrangian-index. Here we just consider the case $L_{0}=\{0\} \bigoplus \mathbb{R}^{n}$.

Proposition 3.11 (Symplectic additivity). For two paths $\gamma_{j} \in \mathcal{P}\left(2 n_{j}\right), j=1,2$, with $n_{1}+n_{2}=n$, there holds

$$
i_{L_{0}}^{L_{1}}\left(\gamma_{1} \diamond \gamma_{2}\right)=i_{L_{0}^{\prime}}^{L_{1}^{\prime}}\left(\gamma_{1}\right)+i_{L_{0}^{\prime \prime}}^{L_{1 \prime}^{\prime \prime}}\left(\gamma_{2}\right)
$$

where $L_{0}=L_{0}^{\prime} \oplus L_{0}^{\prime \prime}$ with $L_{0}^{\prime}=\{0\} \bigoplus \mathbb{R}^{n_{1}}$ and $L_{0}^{\prime \prime}=\{0\} \bigoplus \mathbb{R}^{n_{2}}$, and $L_{1}=$ $L_{1}^{\prime} \oplus L_{1}^{\prime \prime}=P_{1} L_{0}^{\prime} \oplus P_{2} L_{0}^{\prime \prime}$ for some suitable orthogonal symplectic matrixes $P_{1}$ and $P_{2}$.

Proof. By the setup of Subsection 3.1, we can write

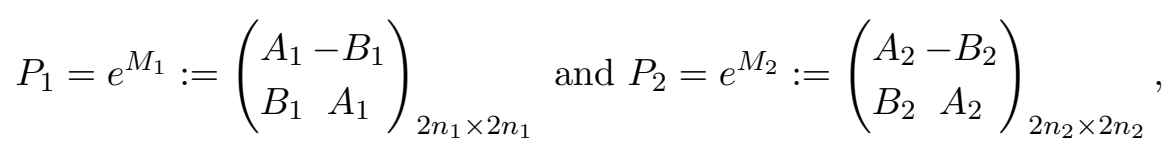

which are both orthogonal symplectic matrices.

Let $\tilde{\gamma}_{1}(t)=e^{-t M_{1}} \gamma_{1}(t)$ and $\tilde{\gamma}_{2}(t)=e^{-t M_{2}} \gamma_{2}(t)$. Then from our definition of the $\left(L_{0}, L_{1}\right)$-index, we have $i_{L_{0}}^{L_{1}}\left(\gamma_{1}(t)\right)=i_{L_{0}}\left(\tilde{\gamma}_{1}(t)\right)$ and $i_{L_{0}}^{L_{1}}\left(\gamma_{2}(t)\right)=i_{L_{0}}\left(\tilde{\gamma}_{2}(t)\right)$. As before, we denote by $\gamma_{1}(t)$ and $\gamma_{2}(t)$ two symplectic matrices of the square block form, that is, $\gamma_{i}(t)=\left(\begin{array}{l}S_{i}(t) V_{i}(t) \\ T_{i}(t) U_{i}(t)\end{array}\right)_{2 n_{i} \times 2 n_{i}}$ for $i=1,2$. 
Then by direct computation, we see that

$$
\begin{aligned}
& \tilde{\gamma}_{1} \diamond \tilde{\gamma}_{2} \\
& =\left(e^{-t M_{1}} \gamma_{1}(t)\right) \diamond\left(e^{-t M_{2}} \gamma_{2}(t)\right)
\end{aligned}
$$

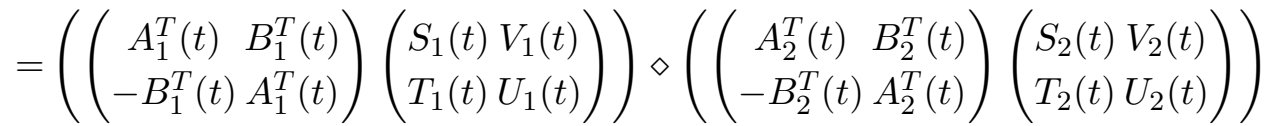

$$
\begin{aligned}
& =\left(\left(\begin{array}{cc}
A_{1}^{T}(t) & B_{1}^{T}(t) \\
-B_{1}^{T}(t) & A_{1}^{T}(t)
\end{array}\right) \diamond\left(\begin{array}{cc}
A_{2}^{T}(t) & B_{2}^{T}(t) \\
-B_{2}^{T}(t) & A_{2}^{T}(t)
\end{array}\right)\right)\left(\left(\begin{array}{c}
S_{1}(t) V_{1}(t) \\
T_{1}(t) U_{1}(t)
\end{array}\right) \diamond\left(\begin{array}{c}
S_{2}(t) V_{2}(t) \\
T_{2}(t) U_{2}(t)
\end{array}\right)\right) \\
& =\left(e^{-t M_{1}} \diamond e^{-t M_{2}}\right)\left(\gamma_{1} \diamond \gamma_{2}\right) \text {. }
\end{aligned}
$$

Hence the definition of $\left(L_{0}, L_{1}\right)$-index implies $i_{L_{0}}^{L_{1}}\left(\gamma_{1} \diamond \gamma_{2}\right)=i_{L_{0}}\left(\tilde{\gamma}_{1} \diamond \tilde{\gamma}_{2}\right)$.

Proposition 3.12 (Homotopy invariant). For two path $\gamma_{j} \in \mathcal{P}(2 n), j=0,1$, it holds that if $\gamma_{0} \sim_{L_{0}}^{L_{1}} \gamma_{1}$, then

$$
i_{L_{0}}^{L_{1}}\left(\gamma_{0}\right)=i_{L_{0}}^{L_{1}}\left(\gamma_{1}\right)
$$

where $\gamma_{0} \sim_{L_{0}}^{L_{1}} \gamma_{1}$ means that there is a map $\delta:[0,1] \rightarrow \mathcal{P}(2 n)$ such that $\delta(j)=\gamma_{j}$ for $j=0,1$ and $v_{L_{0}}^{L_{1}}(\delta(s))$ is constant for $s \in[0,1]$.

Proof. Since $\gamma_{0} \sim_{L_{0}}^{L_{1}} \gamma_{1}$ means that $e^{-t M} \gamma_{0} \sim_{L_{0}} e^{-t M} \gamma_{1}$ due to the definition of the nullity, we can prove the result just by Theorem 3.10 (iv).

Proposition 3.13. For any symplectic path $\gamma \in \mathcal{P}(2 n)$, there holds

$$
i_{L_{0}}^{L_{1}}(\gamma)=i_{L_{0}}\left(e^{-t M} \gamma\right)=\sum_{j=1}^{n} E\left(\frac{\tilde{\theta}_{j}(1)-\tilde{\theta}_{j}(0)}{2 \pi}\right)
$$

where

$$
E(a)=\max \{k \in \mathbb{Z} \mid k<a\},
$$

and $\tilde{\lambda}_{j}(t)=e^{\sqrt{-1} \tilde{\theta}_{j}(t)}$ are the eigenvalues of the matrix

$$
\tilde{\Im}(t)=[\tilde{V}(t)-\sqrt{-1} \tilde{U}(t)][\tilde{V}(t)+\sqrt{-1} \tilde{U}(t)],
$$

where $\tilde{V}(t)=A^{T}(t) V(t)+B^{T}(t) U(t)$ and $\left.\tilde{U}(t)=-B^{T}(t) V(t)+A^{T}(t) U(t)\right)$. Here $e^{t M}=\left(\begin{array}{l}A(t)-B(t) \\ B(t) A(t)\end{array}\right)$ and $\gamma(t)=\left(\begin{array}{l}S(t) V(t) \\ T(t) U(t)\end{array}\right)$. 
Proof. By a direct calculation we have

$$
e^{-t M} \gamma(t)=\left(\begin{array}{ll}
A^{T}(t) & B^{T}(t) \\
-B^{T}(t) & A^{T}(t)
\end{array}\right)\left(\begin{array}{l}
S(t) V(t) \\
T(t) U(t)
\end{array}\right)=\left(\begin{array}{c}
* \tilde{V}(t) \\
* \tilde{U}(t)
\end{array}\right)
$$

and thus by the definition of our new index and Theorem $3.10(\mathrm{v})$, we get the desired result.

3.3. The relation between the $\left(L_{0}, L_{1}\right)$-index and spectral flow. In this section, we will generalize our main Theorem 2.16 to establish the relation between the $\left(L_{0}, L_{1}\right)$-index and spectral flow by an explicit formula.

For convenience, we first introduce some notations about complex symplectic theory as follows. Let $J=\left(\begin{array}{cc}0 & -I_{n} \\ I_{n} & 0\end{array}\right)$ be the standard (real) symplectic matrix defined as before and

$$
\tilde{J}=\left(\begin{array}{cc}
\sqrt{-1} I_{n_{1}} & 0 \\
0 & -\sqrt{-1} I_{n_{2}}
\end{array}\right) \text { with } n_{1}+n_{2}=2 n .
$$

If $n_{1}=n_{2}=n$, then $\tilde{J}$ is called the standard $\tilde{J}$-symplectic matrix on $\mathbb{C}^{2 n}$ and is denoted by $\tilde{J}_{0}$. Recall that the $\tilde{J}$-symplectic group

$$
S p(\tilde{J}) \triangleq\left\{M \mid M^{*} \tilde{J} M=\tilde{J}, M \in \mathcal{L}\left(\mathbb{C}^{2 n}\right)\right\} .
$$

Now consider the standard complex symplectic space $\left(\mathbb{C}^{2 n}, \tilde{\omega}\right)$ with $\tilde{\omega}$ defined

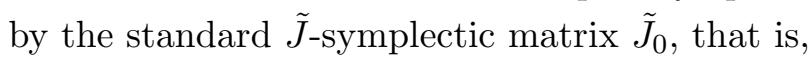

$$
\tilde{\omega}(u, v)=\left\langle\tilde{J}_{0} u, v\right\rangle_{\mathbb{C}}, \forall u, v \in \mathbb{C}^{2 n},
$$

where $\langle u, v\rangle_{\mathbb{C}}$ denotes the standard complex inner products of $u$ and $v$, that is, $u^{*} v$. Denote by $\operatorname{Lag}\left(\tilde{J}_{0}\right)$ the corresponding complex Lagrangian subspaces of $\left(\mathbb{C}^{2 n}, \tilde{\omega}\right)$, that is,

$$
\operatorname{Lag}\left(\tilde{J}_{0}\right)=\left\{\Lambda \text { is a complex subspace of } \mathbb{C}^{2 n} \mid \Lambda=\Lambda^{\tilde{\omega}}\right\},
$$

where $\Lambda^{\tilde{\omega}}=\left\{u \in \mathbb{C}^{2 n} \mid \tilde{\omega}(u, v)=0, \forall v \in \Lambda\right\}$. Denote

$$
\tilde{\Lambda}_{0}=\left\{\left(\begin{array}{c}
x \\
-\sqrt{-1} x
\end{array}\right) \mid x \in \mathbb{C}^{n}\right\}
$$


where $\Lambda_{0}=\left\{\left(\begin{array}{l}0 \\ x\end{array}\right) \mid x \in \mathbb{C}^{n}\right\}$. And let $\tilde{\Lambda}_{1} \triangleq P_{1}^{*} \Lambda_{0}$ where $P_{1}=e^{-t M} P$. It is easy to see that $\tilde{\Lambda}_{1} \in \operatorname{Lag}\left(\tilde{J}_{0}\right)$ since $e^{-t M}$ is a unitary $\tilde{J}_{0}$-symplectic matrix and so is $P$. Now we can state our another main result as follows.

Theorem 3.14. For any symplectic path $\gamma \in \mathcal{P}(2 n)$, there holds

$$
i_{L_{0}}^{L_{1}}(\gamma)=i_{\tilde{\Lambda}_{1}, \tilde{\Lambda}_{0}}(\tilde{\gamma})-n,
$$

where the notations follow the remarks above and $\tilde{\gamma}$ denotes $P_{1}^{*} \gamma P_{1}$.

Proof. The proof of this theorem is essentially due to the one of Theorem 2.16.

Write $\gamma(t) \triangleq\left(\begin{array}{c}S(t) V(t) \\ T(t) U(t)\end{array}\right)$ as before. Suppose $\lambda_{j}(t)=e^{2 \sqrt{-1} \theta_{j}(t)}$ are the eigenvalues of $\tilde{Q}_{\gamma}(t)$ for $j=1,2, \cdots, n$, where

$$
\tilde{Q}_{\gamma}(t)=[\tilde{U}(t)-\sqrt{-1} \tilde{V}(t)][\tilde{U}(t)+\sqrt{-1} \tilde{V}(t)]^{-1}
$$

as in (1.2). Using the results and notations in the previous section, we have

$$
\begin{aligned}
& \sum_{j=1}^{n} E\left(\frac{\tilde{\theta}_{j}(1)-\tilde{\theta}_{j}(0)}{\pi}\right) \\
&=-\sum_{j=1}^{n}\left[-\left(\frac{\tilde{\theta}_{j}(1)-\tilde{\theta}_{j}(0)}{\pi}\right)\right]-n \\
&=-\sum_{j=1}^{n}\left(\left[-\frac{\tilde{\theta}_{j}(1)}{\pi}\right]-\left[-\frac{\tilde{\theta}_{j}(0)}{\pi}\right]\right)-n \\
&=-s f\left\{\left((\tilde{U}(t)-\sqrt{-1} \tilde{V}(t))(\tilde{U}(t)+\sqrt{-1} \tilde{V}(t))^{-1}\right)^{-1}, t \in[0,1]\right\}-n \\
&= \operatorname{Mas}\left\{\left\{\left(\begin{array}{c}
-\sqrt{-1}(\tilde{U}(t)+\sqrt{-1} \tilde{V}(t))(\tilde{U}(t)-\sqrt{-1} \tilde{V}(t))^{-1} x
\end{array}\right) \mid x \in \mathbb{C}^{n}\right\}, \tilde{\Lambda}_{0}\right\}-n \\
&= \operatorname{Mas}\left\{\left(\begin{array}{c}
I \\
\sqrt{-1} I
\end{array}\right)\left(\begin{array}{l}
A^{T}(t) B^{T}(t) \\
B^{T}(t) A^{T}(t)
\end{array}\right)\left(\begin{array}{l}
S(t) V(t) \\
T(t) U(t)
\end{array}\right)\left\{\left(\begin{array}{l}
0 \\
x
\end{array}\right) \mid x \in \mathbb{C}^{n}\right\}, \tilde{\Lambda}_{0}\right\}-n \\
&= \operatorname{Mas}\left\{P_{1}^{*} \gamma P_{1} P_{1}^{*} \Lambda_{0}, \tilde{\Lambda}_{0}\right\}-n \\
&= \operatorname{Mas}\left\{P_{1}^{*} \gamma P_{1} \tilde{\Lambda}_{1}, \tilde{\Lambda}_{0}\right\}-n \\
&= \operatorname{Mas}\left\{\tilde{\gamma} \tilde{\Lambda}_{1}, \tilde{\Lambda}_{0}\right\}-n, \\
& \text { where } \tilde{\gamma}:=P_{1}^{*} \gamma P_{1} \in C\left([0,1], S p\left(\tilde{J}_{0}\right)\right) \text { is applied in the last equality. }
\end{aligned}
$$




\section{AcKnowledgement}

Both authors would like to thank their former common advisor Professor Chungen Liu, for his constant help and encourage in many years. And they also would like to express their deep gratitude to their teachers Professors Yiming Long for his wonderful lectures on the index theory and Chaofeng Zhu for sharing selflessly many critical ideas on this paper. Moreover, the first author is grateful to his present advisor Professor Kefeng Liu for his constant encouragement. Lastly, but not least, they also thank Qingye Zhang, Dong Zhang, Qi Wang and other classmates for many useful discussions on this paper.

\section{REFERENCES}

[1] V. I. Arnold, On a characteristic class entering into the quantization condition, Funkt. Anal. Prilozh. 1 (1967) 1-14 (in Russian) Funct. Anal. Appl. 1 (1967) 1-13 (English trans.)

[2] M. F. Atiyah, V. K. Patodi and I. M. Singer, Spectral asymmetry and Riemannian geometry, Math. Proc. Camb. Phil. Soc., Part I, 77 (1975) 43-69; Part II,78 (1975) 405-432; Part III, 79 (1976) 71-99.

[3] S. E. Cappell, R. Lee and E. Y. Miller, On the Maslov-type index, Comm. Pure Appl. Math. 47 (1994) 121-186.

[4] J. J. Duistermaat, On the Morse index in variational calculus, Adv. Math. 21 (1976) 173-195.

[5] T. Kato, Perturbation theory for linear operators, Springer, Berlin, 1976.

[6] C. Liu, Maslov-type index theory for symplectic paths with Lagrangian boundary conditions, Adv. Nonlinear Stud, 7 (2007), no.1 131-161.

[7] C. Liu, Asymptotically linear Hamiltonian systems with Lagrangian boundary conditions, Pacific J. Math. 232 (2007), no. 1, 233-255.

[8] C. Liu, Q. Wang and X. Lin, An index theory for symplectic paths associated with two Lagrangian subspaces with applications, Nonlinearity 24 (2011) 43-70.

[9] C. Liu and D. Zhang, Iteration theory of Maslov-type index associated with a Lagrangian subspace for symplectic paths and Multiplicity of brake orbits in bounded convex symmetric domains, arXiv:0908.0021.

[10] Y. Long, Index Theory for Symplectic Paths with Applications, Birkhäuser, Basel, 2002.

[11] Y. Long, D. Zhang and C. Zhu, Multiple brake orbits in bounded convex symmetric domain, Adv. in Math. 203 (2006) 568-635.

[12] Y. Long and C. Zhu, Maslov-type index theory for symplectic paths and spectral flow(II), Chinese Ann. Math. 21B (1) (2000) 89-108.

[13] D. McDuff and D. Salamon, Introduction to symplectic topology, Clarendon Press, Oxford, 1998.

[14] J. Robbin and D. Salamon, The Maslov index for paths, Topology 32, 1993, 827-844.

[15] J. Robbin and D. Salamon, The spectral flow and the Maslov index, Bull.London Math.Soc 27, 1995, 1-33. 
[16] C. Zhu, A generalized Morse index theorem. Analysis, geometry and topology of elliptic operators, 493-540, World Sci. Publ., Hackensack, NJ, 2006.

[17] C. Zhu and Y. Long, Maslov-type index theory for symplectic paths and spectral flow (I), Chinese Ann. Math. 20B (4) (1999) 413-424.

Sheng Rao

Center of Mathematical Sciences,

Zhejiang University,

Hangzhou 310027, China

E-mail: rsxiaotang@cms.zju.edu.cn, likeanyone@zju.edu.cn

\section{Xing Lin}

College of Mathematics and Information engineering,

Jiaxing University,

Jiaxing, Zhejiang 314001, China

E-mail: lingauss018@163.com 